\title{
Initial water impact of a wedge at vertical and oblique angles
}

\author{
C. JUDGE*, A. TROESCH and M. PERLIN \\ Department of Naval Architecture and Marine Engineering, University of Michigan, Ann Arbor, MI 48109, USA
}

Received 2 January 2003; accepted in revised form 28 July 2003

\begin{abstract}
This paper examines initial asymmetric wedge-impact flows with horizontal as well as vertical impact velocity. The method of two-dimensional vortex distributions is employed to model the initial-boundary-value problem. The numerical analysis involves discretization of the body surface and an iterative solution technique. Experimental drop tests of a prismatic wedge were performed to gain understanding and provide data for comparison of initial water impact when asymmetry and horizontal impact velocity are present. The experimental investigation of initial flow separation off the wedge vertex (i.e., keel) during impact is described. Initial separation-ventilation of the flow from the vertex due to asymmetric impact or horizontal-vertical impact velocity is examined in relation to the present theory. Agreement between the data and the numerical predictions was demonstrated for small degrees of asymmetry and small ratios of horizontal to vertical impact velocity. The initial flow detachment from the vertex also revealed interesting hydrodynamic characteristics.
\end{abstract}

Key words: asymmetric wedge impact, oblique entry, vortex distributions

\section{Introduction}

High-speed planing boats are widely popular, but little is understood about their stability at high speeds where a significant number of these craft are known to experience unexpected behavior. Research at the University of Michigan intending to understand dynamic instability has included a water-impact model to determine the flow over a cross-section of the hull. This first step towards developing a more complete dynamic model approximates the steady planing of symmetric and asymmetric hulls with constant forward and transverse velocities. The work presented here would be appropriate for determining the restoring forces and moments, i.e., stiffness terms in the equations of motion, in a more general dynamic model. By using a low-order strip theory based upon slenderness assumptions (sometimes referred to as a 2-1/2 D solution) and viewing the planing hull as a series of cross-sections at different points of impact (near the bow the hull is just starting to enter the water while near the transom the hull has mostly entered the water), the model approximates the transverse flow characteristics over the entire hull. The resulting boundary-value problem with appropriate simplifications can be solved numerically using two-dimensional vortex distributions.

This paper examines one aspect of the model: asymmetric wedge-impact flows with combined vertical and horizontal impact velocities. Included in the flow types is initially ventilated flow due to asymmetry and/or horizontal impact velocity. The general model provides initial conditions to start arbitrary, time-dependent, impact simulations [1]. The numerical process involves discretization of the surfaces and an iterative solution technique. The method of two-dimensional vortex distributions is employed to model the boundary-value problem. Experimental investigation into initial flow separation-ventilation off the wedge vertex (i.e., keel)

\footnotetext{
*Presently at the Department of Naval Architecture and Ocean Engineering, United States Naval Academy.
} 
during impact, including asymmetry and horizontal impact velocity, is described. Comparisons between the present model and experimental results are presented.

\section{Problem description}

\subsection{LITERATURE REVIEW}

Early studies on symmetric water impact focused on analysis of seaplane landing. In 1929 von Kármán provided the first theoretical solution for determining the bottom pressures on the pontoon of a landing seaplane [2]. Wagner [3] then extended the expanding flat-plate model of von Kármán to calculate the local water surface elevation. Four decades of subsequent research produced impact models of increasing complexity. See, for example, [4-7]. In the special case of a wedge entering the water with constant velocity, the flow can be assumed to be self-similar. For large impact velocities, or for small time, previous authors have felt it reasonable to expect similarity since the fluid particles near the wedge experience accelerations much larger than gravity, especially in the early stages of water entry. Therefore, gravity has typically been assumed unimportant when the time duration is small or the impact velocity is large. In addition, incompressibility in these earlier works was also assumed.

More recently through the use of matched asymptotic expansions, Cointe [8-10] extended von Kármán's [2] and Wagner's [3] two-dimensional theories for the vertical entry of a horizontal circular cylinder and included the creation of a jet at the intersection of the cylinder and free surface. They found the general simplifying assumptions of incompressible and irrotational flow, an inviscid fluid, a rigid body, and no surface tension or gravity allowed for an accurate prediction of the impact force. Howison et al. [11] solved the two-dimensional water entry problem for sections of small (deadrise) angles by extending the asymptotic expansion methods of Cointe et al. [8] and Wagner [3]. When the impacting body is nearly parallel to the undisturbed water surface, the impacting body is considered to have small deadrise, i.e., the angle between the undisturbed water surface and the body is small.

The assumptions of zero gravity, incompressibility and zero viscosity have also been examined. Greenhow [12] solved the two-dimensional water entry problem including the effects of gravity. Greenhow's calculations found that except perhaps in the jet region, gravity could be ignored if the time was less than the impact velocity divided by twice gravity, i.e., $t \leq$ $v / 2 g$. Zhao and his colleagues $[13,14]$ developed a numerical method, based on a nonlinear boundary-element method, for studying the water entry of a two-dimensional body with arbitrary cross-section. Among their findings, they determined that no air cushion is created under the body during impact as long as the angle between the body and the free surface is larger than $2^{\circ}$ to $3^{\circ}$. Muzaferija et al. [15] included the effects of viscosity in free-surface flows associated with water entry. Viscosity was important for determining the free-surface deformation after the body knuckles (where the body contour turns sharply away from the water surface, see Figure 1) are wetted, but predictions of forces and pressures were very close to those predicted by inviscid theory. They used a finite-volume method to capture the free surface for both two- and three-dimensional water impacts. Various authors have included the effects of compressibility on the flow during impact (e.g. Korobkin [16, 17] or Campana et al. [18]). Compressibility is found to be very important in the first instants as the flow is accelerated from rest. This is particularly true for flat or nearly flat bodies. After the fluid particles have reached the velocities associated with the subsonic stage of water entry, compressibility can be ignored. 
Most investigations of water entry have been restricted to purely vertical impact. By comparison, little progress has been made on understanding oblique entry and its related problem: heeled or yawed planing hulls ${ }^{1}$. In theoretical developments of rounded body impact, Korobkin [20] solved the oblique entry of a blunt symmetric two-dimensional profile and Miloh [21] extended the analytic solution of the vertical impact of a sphere to the case of oblique entry. Of particular relevance to the results presented here, Garabedian [22], Chekin [23], Iafrati [24], and de Divitiis and de Socio [25] completed theoretical and numerical analyses of oblique entry, asymmetric wedge impact. Garabedian [22] and Chekin [23] developed similarity flow models, the later based upon the work of Dobrovol'skaya [7]. Garabedian [22] considered only examples where the vertex of the wedge became the center of similarity. Under this restriction, a skipping motion (i.e., horizontal velocity only) is the sole instance for separationventilation from the vertex. All other impact angles will have both sides wetted [22]. Chekin [23] considered more general separation-ventilation conditions and concluded that there was only one unique combination of wedge angle and impact angle from which no separation of flow from the vertex would occur. For a given wedge and wedge orientation, any other impact angle would force separation. Iafrati [24] adapted a variation of the impact model described by Zhao and Faltinsen [13] and determined pressures and forces for various wedge asymmetries. However, none of his examples lead to flow separation-ventilation from the wedge vertex. de Divitiis and de Socio [25] used a conformal mapping transformation for wedge-shaped bodies with both symmetrical and unsymmetrical entrance velocities. They presented numerical values for the conditions of the onset of flow separation from the wedge vertex. A discussion of their results relative to the work presented here will be given in Section 3.4.

The genesis of the model described in this paper started with Tulin [26]. He solved a contrived three-dimensional planing flow problem in terms of a two-dimensional problem in the cross-flow plane using assumptions of flat bottom, slenderness and small trim angle, i.e., the hull rides at a transom draft and trim placing the waterline entry precisely at the bow apex. This solution allowed for the prediction of the general features of the flow including the pressure distribution on the surface bottom and the location of the spray plume just outboard of the leading edges. Tulin solved this two-dimensional problem using a vortex distribution. Vorus $[27,28]$ extended Tulin's model to wedge impact analysis and introduced a "flat" cylinder theory to include arbitrary sectional-contour impact by means of a physically consistent reordering of variables at the first order. Xu et al. [29] developed a geometrically asymmetric hydrodynamic model for wedge impact that was a complement to Vorus's theory. Savander et al. [30] further extended the usefulness of Vorus's model by incorporating it into a consistent slender body theory capable of modeling large longitudinal variations in planing hull geometry.

Xu's asymmetric model [31], like Vorus's model, considers only vertical impact, i.e., the horizontal velocity component of impact is zero. In addition to the vertical impact velocity and possible asymmetry, the model presented here allows the body to travel in the horizontal direction before and during impact. A symmetric body impacting with horizontal velocity will produce a flow similar to an asymmetric impact with only vertical velocity when rotation about the $x$-axis is not allowed. The horizontal component of the impact velocity causes the

\footnotetext{
${ }^{1}$ With regard to planing hulls, Savitsky et al. [19] conducted an experimental study on flat plate and twenty degree deadrise models subject to non-zero heel and yaw angles. These experiments will provide a valuable force and moment data base when the asymmetric impact models are incorporated into an asymmetric planing hull theory.
} 
flow to move along the contour faster on one side than on the other, creating hydrodynamically asymmetric impact.

\subsection{THEORETICAL-MODEL BACKGROUND}

The two-dimensional theory of Vorus [28] exploits the flatness of the section contour where the transverse flow perturbation velocity tends toward infinity at the intersection points. As the two-dimensional cylinder bottom tends toward horizontal, boundary conditions along the horizontal axis apply with increasing accuracy, implying a limit of geometric linearity. However, the increase in transverse flow perturbation velocity resulting from this flatness implies increasing hydrodynamic nonlinearity. Vorus's method represents a compromise theory. It is geometrically linear, in that the flat cylinder boundary conditions are applied on the horizontal axis, but it is hydrodynamically nonlinear and so retains the large perturbation flow produced by the impacting flat cylinder. Assumptions of zero gravity, incompressibility and zero viscosity are used to simplify the water impact model. These effects are believed to be small.

While the theory of Vorus was developed as an asymptotic solution valid for diminishing deadrise, it has been evaluated at significantly larger angles. For constant impact velocity, the theory yields a similarity solution and as such, is readily comparable to the various models developed since von Kármán. For example, Figure 10 of Vorus [28] and Figure 12 of Savander et al. [30] show the two-dimensional wedge impact added-mass coefficients as a function of deadrise angle, $\beta$. When normalized by the square of the tangent of $\beta$, the classic theories of von Kármán and Wagner produce constant added mass values, independent of $\beta$. For angles up to approximately 35-40 degrees, the various theories are bounded by these values: Wagner as an upper bound and von Kármán as a lower one. The theories of Sydow [4], Mayo [5], Blisplinghoff and Dohery [6], Dobrovol'skaya [7], Zhao and Faltinsen [13], Vorus [28], Fontaine and Cointe [32], and Savander et al. [30] appear to asymptote to Wagner's solution in the limit of zero deadrise and, though at different rates, approach von Kármán's for $\beta$ greater than 35 degrees. The theoretical impact coefficients of Vorus and Savander are consistently less than those of Sydow, Blisplinghoff and Dohery, Dobrovol'skaya, Zhao and Faltinsen, and Fontaine and Cointe but higher than that of Mayo. However, when the theoretical accelerations of a twenty degree impacting wedge are compared with experiments conducted by Blisplinghoff and Dohery [6] or Peterson et al. [33], the predictions track closer to the accelerations, underpredicting the maximum impact acceleration by less than 5\% [30, Figures 13, 14]. The other theories produce an overprediction of the impact acceleration/force. The basic impact models of Tulin [26] and Vorus [28] sacrifice some of the free-surface details of the jet flow past the body's zero pressure point. However, as previous experiments have demonstrated and as the experiments described in this paper demonstrate, this is not a serious drawback when considering the flow field next to the body.

To begin the analysis, a body-fixed coordinate system is used with $y$ and $z$ representing the horizontal and vertical axes, respectively. The body geometry is known and a small elevation (i.e., deadrise) angle, $\beta(y)$, is assumed (see Figure 1). The pre-impact vertical downward velocity, $W$, and horizontal velocity, $U$, of the cylinder are prescribed. $Z_{W L}(t)$ represents the height of the undisturbed water surface above the vertex (point $O$ ), see Figure 1, where

$$
Z_{W L}(t)=\int_{0}^{t} \mathrm{Wd} \tau
$$




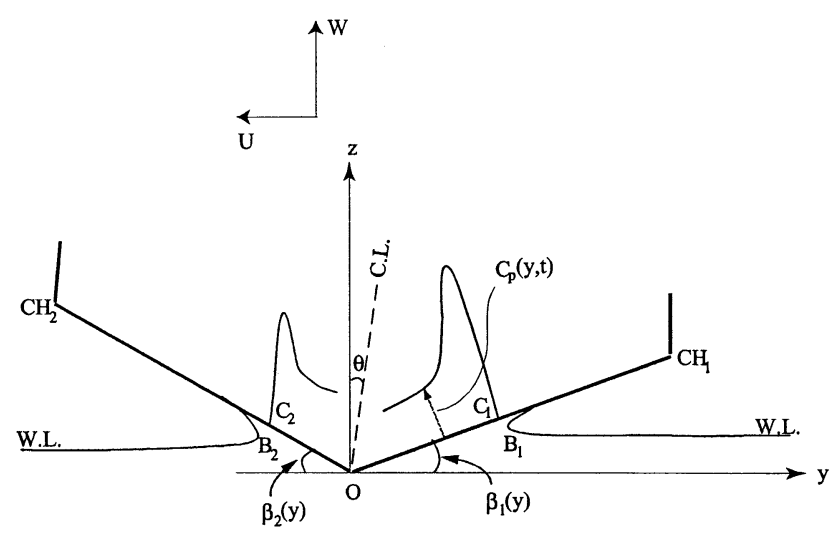

Figure 1. Definition sketch of Type A model of cylinder asymmetric impact (small asymmetry) and horizontal impact velocity. $U, W$ are equivalent free-stream velocities.

On impact, the free surface advances on each side upward along the body contour and forms initially attached jets, as shown in Figure 1. Point $B$ may be called the jet "sprayroot" and travels rapidly outward along the cylinder contour. $B$ is followed closely by $C$, the "zero-pressure" point. For $y$-coordinate greater than $C$ the dynamic pressure is zero. While $B$ is attached to the contour, the pressure has a sharp spike with a large negative gradient toward $C$. The large pressure gradient is a result of the large flow acceleration near $C$. As time advances, both $B$ and $C$ move outward. The models of Tulin [26] and Vorus [28] have a jump in transverse velocity at $B$ (from the upper branch of the free-surface streamline to the lower). However, the potential is continuous at $B$ as is the displacement, by displacement continuity.

For asymmetric flows, due to either geometric asymmetry $\left(\beta_{1} \neq \beta_{2}\right)$ or kinematic asymmetry $(U \neq 0)$ points $B$ and $C$ are different for each side. They are identified as $\left(B_{1}, C_{1}\right)$ and $\left(B_{2}, C_{2}\right)$. The subscripts 1 and 2 represent the positive $y$ and negative $y$ coordinates, respectively. The horizontal coordinates of the jet spray-roots are $Y_{B 1}(t)$ and $Y_{B 2}(t)$ and the horizontal coordinates of the zero-pressure points are $Y_{C_{1}}(t)$ and $Y_{C 2}(t)$. The zero-pressure points, $C_{1}$ and $C_{2}$, are also called the wetted points. The wetted surface with non-zero dynamic pressure, i.e., non-zero $C_{p}$, is defined between the wetted points. $C_{p}$ is the hydrodynamic pressure coefficient:

$$
C_{p}=\frac{\left(p-p_{\infty}\right)}{\frac{1}{2} \rho W_{o}^{2}}
$$

where $\rho$ is the fluid density, $p$ is the local total pressure, $p_{\infty}$ is atmospheric pressure, and $W_{o}$ is the initial vertical impact velocity of the body.

Without loss of generality, $\beta_{1} \leq \beta_{2}$ is assumed. The so-called angle of heel representing the geometric asymmetry is defined by

$$
\theta=\frac{\beta_{2}\left(0^{-}\right)-\beta_{1}\left(0^{+}\right)}{2},
$$

where $\beta_{1}\left(0^{+}\right)$and $\beta_{2}\left(0^{-}\right)$are the elevation (i.e., deadrise) angles at the vertex on the right and left sides, respectively. Vorus [28], Xu [31], and Judge [1] consider $\beta$ as a function of $y$, i.e., variable deadrise. For the work presented here, $\beta$ is a constant, independent of $y$.

$\mathrm{Xu}$ [31] and $\mathrm{Xu}$ et al. [29] defined two types of asymmetric impact. Type A flow, Figure 1, is when there is small asymmetry and the flow moves outward along the contour on both 


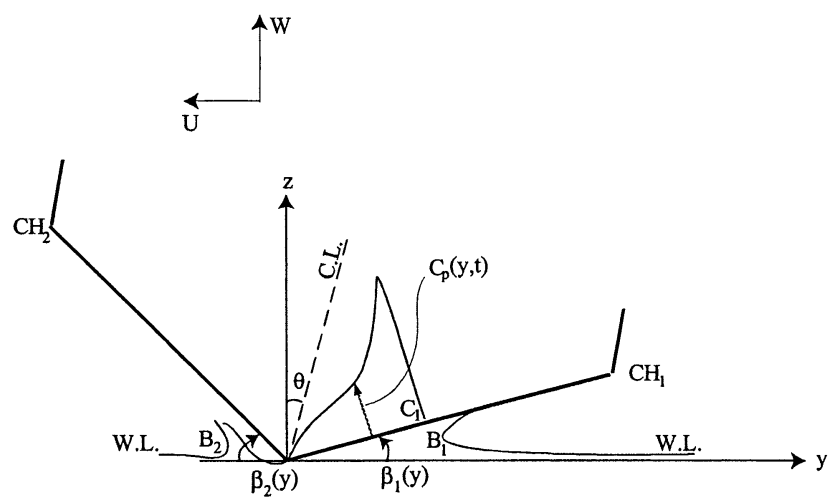

Figure 2. Type B model of cylinder asymmetric impact (large asymmetry) and horizontal impact velocity. $U, W$ are equivalent free-stream velocities.

sides of the vertex. Type B flow, Figure 2, occurs when there is large asymmetry and the flow detaches from the body contour at the vertex on one side.

The elevation (i.e., deadrise) angle $\beta_{1}$ is assumed small and characterizes the bottom flatness. By definition, Type A flow always attaches to the bottom before moving along the contour. If $\theta \rightarrow 0$ and $U \rightarrow 0$, the limiting behavior of the asymmetric impact flow approaches the corresponding symmetric solution derived by Vorus [28]. The onset of Type B flow is calculated from the initial positions of the zero-pressure points $\left(C_{1}\right.$ and $\left.C_{2}\right)$ for different $\theta$ and $U / W$. As $\theta$ or $U / W$ increases, $C_{2}$ moves closer towards the vertex. When $C_{2}$ reaches the vertex, the jet flow on the left side is assumed to detach. The impact flow of Type $A$ then is identified as Type B impact flow. The Type A model includes an infinite velocity at the sharp vertex. This singularity generally exists for any asymmetric flow. Using numerical studies, $\mathrm{Xu}$ [31] explains how the flow separates at the keel due to asymmetry and then quickly re-attaches to the bottom for small asymmetric impact. This implies that a cavity flow due to water-entry is limited to a very small region for Type A impact and not included in the impact model presented here.

Vorus [28], $\mathrm{Xu}$ [31], and the present model use a small deadrise angle approximation in the solution method. However, there is a difference in where the approximation is made. Both models define the vertical perturbation velocity from the strength of the vortices placed on the contour. Vorus [28] and $\mathrm{Xu}$ [31] assume the tangential perturbation velocity on the body is equal to the velocity generated by the vortices. The model presented in this work defines the horizontal perturbation velocity on the body as the velocity generated from the vortices employing a small angle approximation (a Taylor series expansion in $z$ about $z=0$, the horizontal axis). The details of the expansion are given by Judge [1, Appendix C] and will not be repeated here. In this case, the perturbation horizontal and perturbation vertical velocities are defined by the vortex distribution, $\gamma(\chi, t)$, placed on the $z=0$ boundary and the tangential and normal velocities are determined from a first order Taylor series expansion from the horizontal axis. Specifically, the horizontal and vertical velocity components on the $z=0$ axis are,

$$
\begin{aligned}
& u(\chi, t)=-\frac{1}{2} \gamma(\chi, t), \\
& w(\chi, t)=\frac{1}{2 \pi} \int_{-b_{2}(t)}^{b_{1}(t)} \frac{\gamma(s, t)}{s-\chi} \mathrm{d} s,
\end{aligned}
$$


respectively.

The total normal and tangential velocities on the body contain both the fall velocities and the perturbation velocities in the normal and tangential directions, respectively. Therefore,

$$
\begin{aligned}
& \bar{V}_{n}=V_{n}(\chi, t)+v_{n}(\chi, t), \\
& \bar{V}_{s}=V_{s}(\chi, t)+v_{s}(\chi, t),
\end{aligned}
$$

where $V_{n}$ and $V_{s}$ are the fall velocities, $v_{n}$ and $v_{s}$ are the perturbation velocities and $\bar{V}_{n}$ and $\bar{V}_{s}$ are the total velocities. The $n$ and $s$ subscripts indicate the normal and tangential directions, respectively. The velocities in the normal and tangential directions are made of the vertical and horizontal velocity components, that is,

$$
\begin{aligned}
& V_{n}=W \cos \beta+U \sin \beta, \\
& V_{s}=W \sin \beta-U \cos \beta,
\end{aligned}
$$

and

$$
\begin{aligned}
& v_{n}=w \cos \beta-u \sin \beta, \\
& v_{s}=w \sin \beta+u \cos \beta,
\end{aligned}
$$

where $W$ and $U$ are the fall velocities in the vertical and horizontal directions, respectively, and $w$ and $u$ are the perturbation velocities in the vertical and horizontal directions, respectively.

\section{Oblique impact theoretical formulation}

\subsection{SOLUTION METHOD}

The total normal and tangential velocities along the wedge body contours in terms of the vertical and horizontal velocities are

$$
\begin{aligned}
& \bar{V}_{n}=(W+w) \cos \beta+(U-u) \sin \beta, \\
& \bar{V}_{s}=(W+w) \sin \beta-(U-u) \cos \beta,
\end{aligned}
$$

where $\mathrm{W}$ and $\mathrm{U}$ are the free-stream velocities (in body-fixed coordinates), and $w$ and $u$ are the perturbation velocities due to the hydrodynamic flow field. Due to the different elevation (i.e., deadrise) angles on the two sides,

$$
\beta=\left\{\begin{aligned}
\beta_{1} & \text { if } y>0 \\
-\beta_{2} & \text { if } y<0
\end{aligned}\right.
$$

Figure 3 presents a vector representation of the body boundary conditions. Rolling angular velocity (rotation about the $x$-axis) due to asymmetric impact is considered of higher order and therefore is neglected in the formulation of the body boundary condition. For the formulation and experiments presented here, the wedge is constrained from roll.

A vortex distribution is used to solve the resulting boundary-value problem. Bound vortices are placed on the wetted body surface, between $C_{1}$ and $C_{2}$. The end bound vortices are shed and become free vortices that form jet flows that follow the body contour. A condition of zero tangential velocity outside the jet "spray-roots" $\left(B_{1}\right.$ and $\left.B_{2}\right)$ identically satisfies the condition of atmospheric pressure on the free surface. 


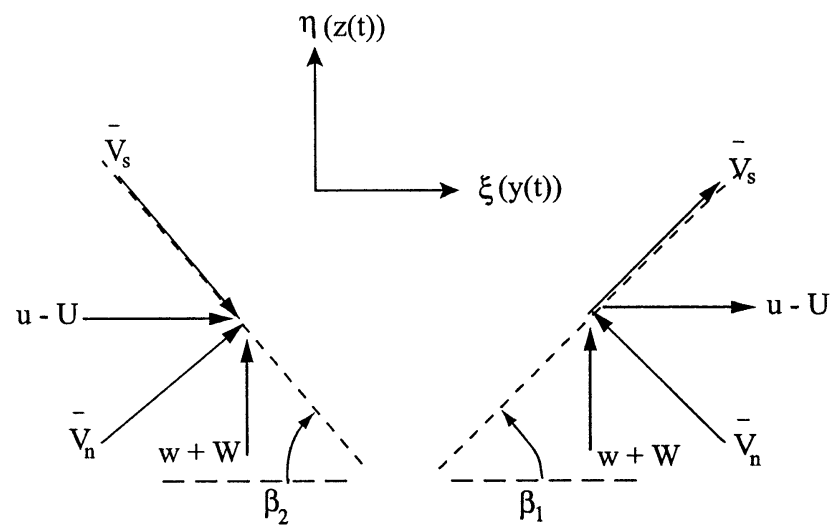

Figure 3. Contour Velocity $-\bar{V}_{s}$ and $\bar{V}_{n}$ are the total tangential and normal velocities on the contour; $\mathrm{W}$ and $\mathrm{U}$ are the free-stream vertical and horizontal velocities, respectively; $w$ and $u$ are the perturbation vertical and horizontal velocities, respectively.

The flatness is exploited by collapsing the body and free-surface contours to a horizontal level for satisfying the boundary conditions. Following Vorus's analysis of the limiting physics of the symmetric problem, the effects of the jets beyond the jet "spray-root" locations, $B_{1}$ and $B_{2}$, are neglected and the lower half-space represents the fluid domain under the body and the free-surface contours. Physically, this means that the horizontal flow beyond points $B_{1}$ and $B_{2}$ is approximated as zero to first order.

The linear dimensions of the solution space are scaled by the positive $y$-axis zero-pressure point location, $C_{1}$. So

$$
\xi=\frac{y(t)}{Y_{C 1}(t)},
$$

where $y$ is the distance along the horizontal axis. The mathematical solution space is shown in Figure 4 (a). The $y$-axis boundary representation is the dashed segments of the complete contour indicated in Figure 4 (b).

The boundary moves from the upper branch at $B_{1}$ and $B_{2}$ to the lower branch with a jump discontinuity in tangential velocity. The nondimensional locations of $C_{1}, B_{1}, C_{2}$ and $B_{2}$ are $c_{1}(t), b_{1}(t),-c_{2}(t)$ and $-b_{2}(t)$, respectively. The total normal and tangential velocities, $\bar{V}_{n}$ and $\bar{V}_{s}$, are scaled by the initial vertical impact velocity, $W_{o}$. As usual, the vortices are arranged on the contour such that they satisfy the boundary conditions. The strength of the vortex distribution is

$$
\gamma(\xi, t)=-2 u(\xi, t),
$$

where $u$ is the perturbation velocity in the horizontal direction.

\subsection{GOVERNING EQUATION AND BOUNDARY CONDITIONS}

The entire fluid region is assumed to be incompressible, irrotational and homogeneous. This problem can then be considered a potential flow problem governed by Laplace's equation,

$$
\nabla^{2} \Phi=0 .
$$

Along with a zero gravity assumption, the following boundary conditions apply to the flow: 


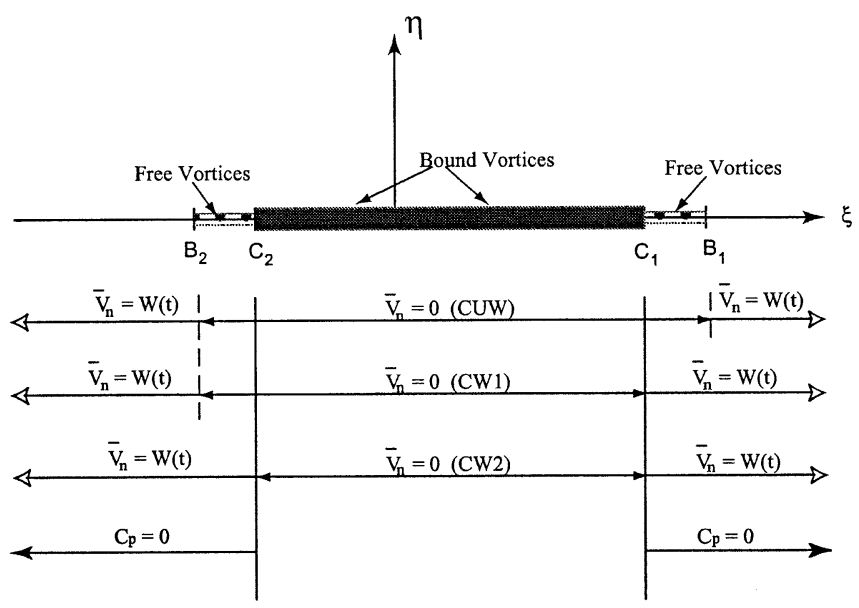

(a). Mathematical Solution Space

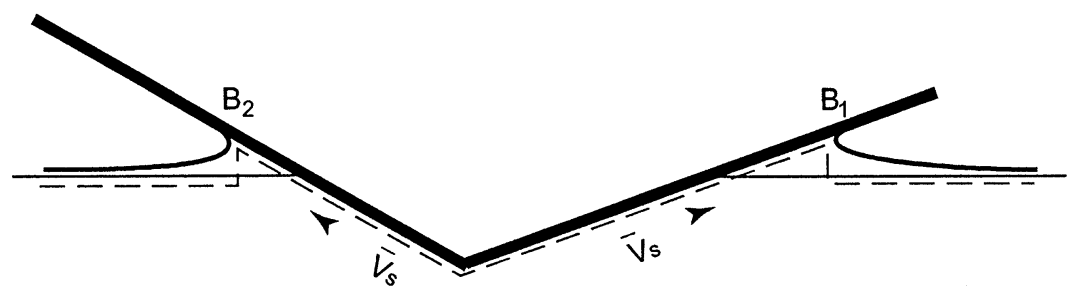

Figure 4. (a) Mathematical model with vortex distributions and boundary conditions, where $\xi$ is the horizontal coordinate nondimensionalized by $Y_{C 1}(t)$. All velocities are scaled by $W_{o}$. "CUW", "CW1", and "CW2" denote chines unwetted, chines wet side 1 , and chines wet both sides, respectively. (b) The physical boundary between the lower and upper mathematical half-spaces. The boundary switches from the upper branch to the lower branch at $B_{1}$ and $B_{2}$ with a jump in tangential velocity.

The far field condition requires that the perturbation velocities go to zero there, i.e.,

$$
\mathbf{V}_{\infty} \rightarrow-\mathrm{U} \hat{\jmath}+\mathrm{W} \hat{k}
$$

where $\mathbf{V}_{\infty}$ is the velocity of the far field flow with respect to the translating reference frame associated with the falling body. $\hat{j}$ denotes the unit vector in the positive horizontal direction, to the right, and $\hat{k}$ denotes the unit vector in the vertically upward direction. The body is falling and moving to the right.

The kinematic boundary conditions generally state that the flow can move only tangentially to the boundary surfaces. Therefore, in the moving coordinate system, the normal velocity to the body must equal zero. On the body then

$$
\bar{V}_{n}=\mathbf{V} \cdot \mathbf{n}=0,
$$

where $\mathbf{V}$ is the velocity on the surface and $\mathbf{n}$ is the normal, positive defined external to the body. The boundary condition (19) is transformed through the application of Equations (12), (4), (5), and (8) into an equation in terms of the vortex distribution:

$$
\frac{1}{2} \gamma_{c}(\xi, t) \sin \beta+\frac{\cos \beta}{2 \pi} \int_{-b_{2}(t)}^{b_{1}(t)} \frac{\gamma(s, t)}{s-\xi} \mathrm{d} s=-V_{n}(\xi, t)
$$


for $-c_{2}(t) \leq \xi \leq 1$, where $\gamma$ represents both the bound vortex distribution, $\gamma_{c}$, between $-c_{2}$ and 1 and the free-vortex distribution, $\gamma_{s}$, between $-b_{2}$ and $-c_{2}$ and between 1 and $b_{1}$. This equation takes the form of a Carleman singular integral equation of the second kind and can be solved to find the strength of the bound-vortex distribution, $\gamma_{c}$, following the solution procedure in Muskhelishvili [34] or Tricomi [35].

The Kutta condition requires that the velocity be continuous and bounded across the zeropressure point locations, $C_{1}$ and $C_{2}$. The singularities in the bound vortex distribution, resulting from the kinematic body boundary condition, must be removed at these points. (See Savander et al. [30] for a lucid explanation of how this relates to Tulin's theory.) The Kutta condition then can be written as follows:

$$
\begin{array}{r}
\frac{1}{2 \pi} \int_{1}^{b_{1}(t)} \frac{\gamma_{s}(s, t) \mathrm{d} s}{\chi(s, t)\left(s+c_{2}(t)\right)(s-1)}-\frac{1}{2 \pi} \int_{-b_{2}(t)}^{-c_{2}(t)} \frac{\gamma_{s}(s, t) \mathrm{d} s}{\chi(s, t)\left(s+c_{2}(t)\right)(s-1)}= \\
-\frac{1}{\pi} \int_{-c_{2}(t)}^{1} \frac{V_{n}(s, t) \mathrm{d} s}{\chi(s, t)\left(s+c_{2}(t)\right)(1-s)}
\end{array}
$$

and

$$
\begin{gathered}
\frac{1}{2 \pi} \int_{1}^{b_{1}(t)} \frac{\gamma_{s}(s, t) s \mathrm{~d} s}{\chi(s, t)\left(s+c_{2}(t)\right)(s-1)}-\frac{1}{2 \pi} \int_{-b_{2}(t)}^{-c_{2}(t)} \frac{\gamma_{s}(s, t) s \mathrm{~d} s}{\chi(s, t)\left(s+c_{2}(t)\right)(s-1)}= \\
-\frac{1}{\pi} \int_{-c_{2}(t)}^{1} \frac{V_{n}(s, t) s \mathrm{~d} s}{\chi(s, t)\left(s+c_{2}(t)\right)(1-s)}+\frac{1}{2 \pi}\left[\int_{1}^{b_{1}(t)} \gamma(s, t) \mathrm{d} s+\int_{-b_{2}(t)}^{-c_{2}(t)} \gamma(s, t) \mathrm{d} s\right],
\end{gathered}
$$

where

$$
\chi(\xi, t)=\frac{\kappa(\xi, t)}{\left(\left|\left(\xi+c_{2}(t)\right)(1-\xi)\right|\right)^{\frac{1}{2}}}
$$

and where $\kappa(\xi, t)$ is defined in [29]. For constant deadrise angles, $\kappa(\xi, t)$ is

$$
\kappa(\xi, t)=|\xi|^{-\frac{\beta_{1}+\beta_{2}}{\pi}}|1-\xi|^{\frac{\beta_{1}}{\pi}}\left|\xi+c_{2}(t)\right|^{\frac{\beta_{2}}{\pi}} .
$$

The dynamic boundary condition requires that, along the unwetted body and the free surface, the pressure must equal atmospheric pressure. So, for $y \geq Y_{C 1}(t)$ and $y \leq Y_{C 2}(t)$,

$$
C_{p}=0 .
$$

This zero pressure requirement yields the velocities of the "jet roots",

$$
\begin{aligned}
Y_{B 1 t} & =\frac{{\overline{V_{s}}}^{2}\left(b_{1}, t\right)-V_{s}^{2}\left(b_{1}, t\right)-V_{n}^{2}\left(b_{1}, t\right)}{2 v_{s}\left(b_{1}, t\right)}, \\
Y_{B 2 t} & =\frac{\bar{V}_{s}^{2}\left(-b_{2}, t\right)-V_{s}^{2}\left(-b_{2}, t\right)-V_{n}^{2}\left(-b_{2}, t\right)}{2 v_{s}\left(-b_{2}, t\right)},
\end{aligned}
$$

where $Y_{B 1 t}=\mathrm{d} Y_{B 1}(t) / \mathrm{d} t$ and $Y_{B 2 t}=\mathrm{d} Y_{B 2}(t) / \mathrm{d} t$ are the time rate-of-change of locations $B_{1}$ and $B_{2} ; \bar{V}_{s}$ is the total tangential velocity; $V_{n}$ and $V_{s}$ are the free-stream velocities in the normal and tangential directions, respectively; and $v_{s}$ is the perturbation velocity in the contour tangential direction. These equations are derived by applying Bernoulli's pressure equation 


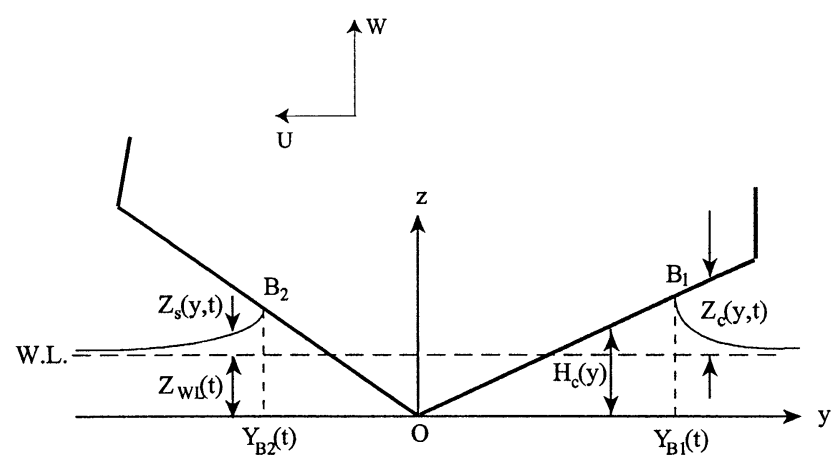

Figure 5. Displacement contour of the body and the free surface.

in a moving reference frame (neglecting gravity) and Equation (25). The full derivation of Equations (26) and (27) is found in [1, Appendix A].

Conservation of mass (or displacement continuity) requires a continuous body-free-surface contour of $-\infty<y<\infty$ to lowest order. The elevation of the free surface must match the height of the body contour at the jet spray-roots, $B_{1}$ and $B_{2}$ (see Figure 5). This matching was argued intuitively by Wagner [3] and formally demonstrated, to second order, by Fontaine and Cointe [36]. The displacement continuity conditions can be written as follows (see [1, Chapter 3.6] for details):

$$
\begin{aligned}
Z_{W L}(t)= & \frac{1}{\pi} \int_{-c_{2}^{*}(t)}^{1} \frac{\cos \beta(s) H_{c}(s)}{\chi^{*}(s, t)\left(s+c_{2}^{*}(t)\right)(1-s)} \mathrm{d} s \\
& -\frac{Y_{H}(t)}{\pi} \int_{-c_{2}^{*}(t)}^{1} \frac{\sin \beta(s)}{\chi^{*}(s, t)\left(s+c_{2}^{*}(t)\right)(1-s)} \mathrm{d} s
\end{aligned}
$$

and

$$
\begin{aligned}
\frac{\beta_{1}}{\pi} & Z_{W L}(t)+\frac{Y_{H}(t)}{2 \pi} \int_{-c_{2}^{*}(t)}^{1} \frac{\sin \beta(s)}{\chi^{*}(s, t)(1-s)} \mathrm{d} s-\frac{1}{2 \pi} \int_{-c_{2}^{*}(t)}^{1} \frac{\cos \beta(s) H_{c}(s)}{\chi^{*}(s, t)(1-s)} \mathrm{d} s= \\
& c_{2}^{*}(t) \frac{\beta_{2}}{\pi} Z_{W L}(t)+\frac{Y_{H}(t)}{2 \pi} \int_{-c_{2}^{*}(t)}^{1} \frac{\sin \beta(s)}{\chi^{*}(s, t)\left(s+c_{2}^{*}(t)\right)} \mathrm{d} s \\
& -\frac{1}{2 \pi} \int_{-c_{2}^{*}(t)}^{1} \frac{\cos \beta(s) H_{c}(s)}{\chi^{*}(s, t)\left(s+c_{2}^{*}(t)\right)} \mathrm{d} s,
\end{aligned}
$$

where

$$
Z_{W L}(t)=\int_{0}^{t} \mathrm{Wd} \tau \quad \text { and } \quad Y_{H}(t)=\int_{0}^{t} \mathrm{Ud} \tau
$$

and where $c_{2}^{*}$ is the ratio of the left-hand-side jet-spray root location to the right-hand-side jet-spray root location,

$$
c_{2}^{*}=-\frac{Y_{B 2}(t)}{Y_{B 1}(t)}
$$

here $\chi^{*}$ is given by Equations (23) and (24) where $c_{2}$ is replaced by $c_{2}^{*}$, and $H_{c}(y)$ is defined in Figure 5. Equation (29) is automatically satisfied for the symmetric case with no horizontal 


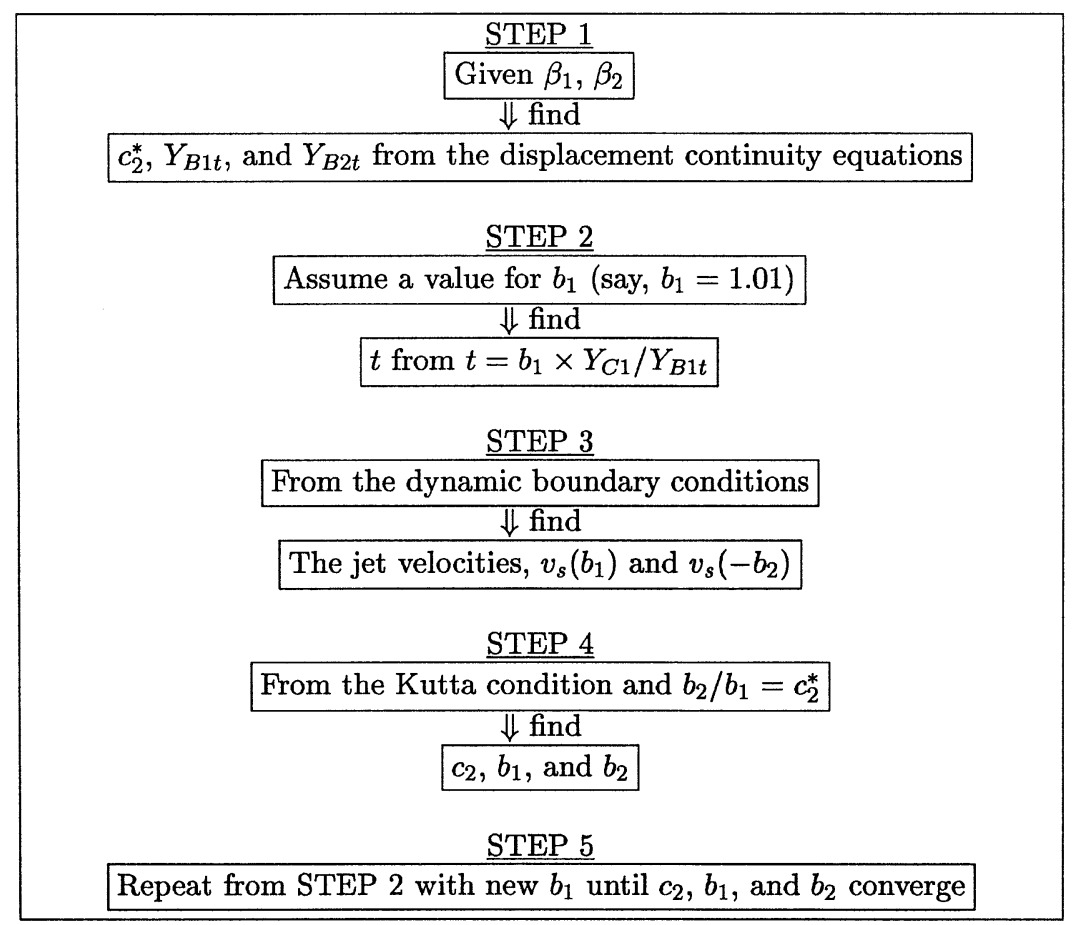

Figure 6. Iterative method to determine initial wetting conditions.

velocity. A more complete discussion of the derivation of the Equations (28) and (29) can be found in [1]. It generally follows the analysis first given by Vorus [27] for symmetric impact and $\mathrm{Xu}$ et al. [29] for asymmetric impact without horizontal velocity.

\subsection{INITIAL WETTING CONDITIONS}

The body contour is assumed to have constant, nonzero elevation (i.e., deadrise) angles, $\beta_{1}$ and $\beta_{2}$, near the vertex. In addition, in the small time after initial impact the velocities of the impact can be taken as constant at the initial values, $\mathrm{W}(0) \equiv \mathrm{W}_{0}$ and $\mathrm{U}(0) \equiv \mathrm{U}_{0}$. All velocity variables are nondimensionalized by $\mathrm{W}_{0}$; the constant vertical impact velocity is set equal to unity and the constant horizontal impact velocity is the ratio of horizontal to vertical impact velocity, $U=U_{0} / W_{0}$. The locations of the zero-pressure points and effective jet "sprayroots" as well as the jet velocities are determined using the boundary condition equations and an iterative method. A flow chart of this process is presented in Figure 6.

Generally, to determine the initial conditions start with the given deadrise angles, $\beta_{1}$ and $\beta_{2}$. Next iterate Equations (28) and (29) (displacement continuity equations) to get $c^{*}, Y_{B 1 t}$, and $Y_{B 2 t}$. Assume an initial iterative value for $b_{1}$ that is greater than one, for example, $b_{1}=1.01$ and then calculate an initial $t$ from $Y_{B 1 t}$ and $t=b_{1} / Y_{B 1 t}$. From Equations (26) and (27) (dynamic boundary equations), calculate the jet velocities, $v_{s}\left(b_{1}\right)$ and $v_{s}\left(-b_{2}\right)$. The nondimensional coordinates $c_{2}, b_{1}$ and $b_{2}$ are re-solved by iterating Equations (21) and (22) (Kutta condition) and using $b_{2} / b_{1}=c_{2}^{*}$. Repeat these steps with the new $b_{1}$ until $c_{2}, b_{1}$ and $b_{2}$ converge. For a more complete description of the numerical implementation of the steps shown in Figure 6, see [1, Chapter 4]. 


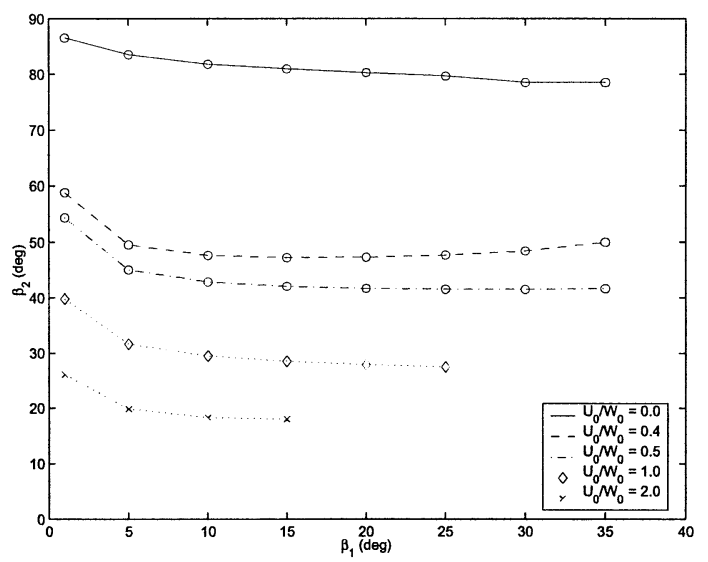

Figure 7. The critical angle, $\beta_{2}$ versus $\beta_{1}$ at which ventilation occurs off the vertex for different ratios of impact velocities. Areas below the curve are Type A flows and areas above the respective curves are Type B flows.

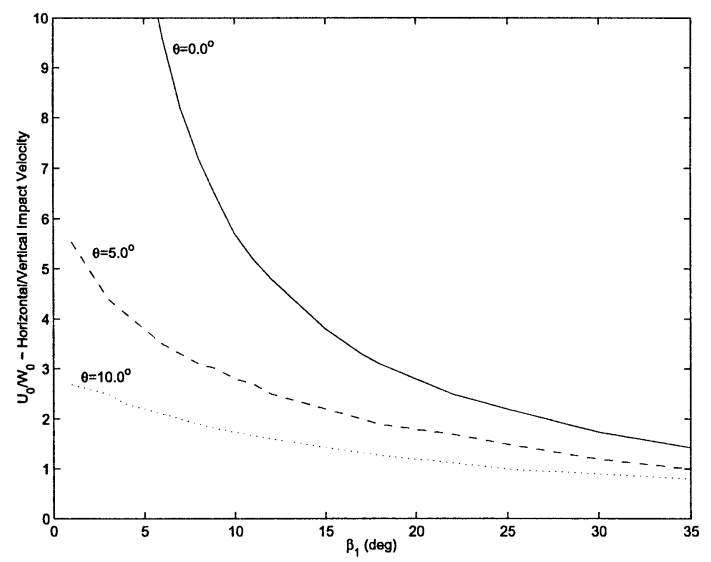

Figure 8 . The critical value of $U_{0} / W_{0}$ versus the corresponding $\beta_{1}$ at which ventilation occurs off the vertex for different angles of $\theta$. Areas below the respective curves are Type A flows and areas above the curves are Type B flows.

\subsection{INITIATION OF TYPE B FLOW}

In a similar manner to the investigation in Xu et al. [29] on vertical impacts, the limits of asymmetric impact with horizontal impact velocity are examined, in particular the transition between Type A and Type B impact. As the geometric asymmetry, $\theta$, or the kinematic asymmetry, $U / W$, increases the zero-pressure point on the left side, $c_{2}$, moves back toward the vertex. When $c_{2}$ reaches the slope discontinuity at the vertex, i.e., $c_{2}=0$, the impact is defined as Type B. The phrases "Type B impact" and "ventilation" are used interchangeably when discussing this limiting behavior. Both terms imply a flow detachment at the wedge vertex that may or may not eventually produce reattachment to the body with an air pocket present ${ }^{2}$. Figure 7 shows the limiting angle of $\beta_{2}$ versus $\beta_{1}$ at which Type $\mathrm{B}$ flow is initiated according to the theory.

Figure 8 shows the critical value of $U_{0} / W_{0}$ versus the angles at which ventilation occurs off the vertex, i.e., Type B impact. For symmetric bodies, i.e., $\theta=0$, and small elevation (i.e., deadrise) angles, the horizontal velocity must be much greater than the vertical velocity for ventilation to occur. However, as the deadrise angle increases the required ratio decreases rapidly and then flattens. Thus, the critical value of $U_{0} / W_{0}$ is less for bodies of larger deadrise angles. Intuitively, this is reasonable.

For a given ratio of horizontal to vertical impact velocity, the $\beta_{2}$ angle required for ventilation can be determined numerically. In Figure 7, the dependence of $\beta_{2}$ on $\beta_{1}$ for the initiation of Type B flow is small. An increase in horizontal velocity decreases the $\beta_{2}$ required for ventilation. Figure 8 shows the critical value of $U_{0} / W_{0}$ versus $\beta_{1}$ for different degrees of

\footnotetext{
${ }^{2}$ Any reattachment would come much later in the impact process and, as such, not be covered by the theory presented here.
} 
asymmetry (as measured by $\theta$ ). As expected, larger degrees of asymmetry reduce the critical ratio of impact velocities.

It is possible to compare results shown in Figures 7 and 8 with the results given by Divitiis and de Socio [25]. Those authors predicted the limiting angle of detachment using a completely different approach - numerical analysis of conformal transformations. While their results did not include a rotated wedge configuration, they did consider a symmetric wedge, i.e., $\beta_{1}=\beta_{2}$ or $\theta=0$, with various "side-slip" entrance velocities. Generally Divitiis and de Socio [25] predict separation much earlier than the results shown in Figure 8 and demonstrated by the experiments presented in the following sections. As an example, in Figure 6 of Divitiis and de Socio [25], a symmetric wedge with a $37^{\circ}$ deadrise angle and zero heel would experience flow separation from one side with a "side slip" ratio of approximately of $U_{0} / W_{0}=0 \cdot 07$. This would correspond to an entrance velocity of $5^{\circ}$ from vertical. In comparison, Figure 8 (theory) and Table 3 (experiment) of this work show vertex ventilation occurring at $U_{0} / W_{0}=1.5$ and $2 \cdot 0$, respectively. These values correspond to entrance velocities of $56^{\circ}$ and $63^{\circ}$, respectively. The significant theoretical differences between the two approaches are likely related to the different ways in which the flow around the vertex is modeled. Divitiis and de Socio[25] impose a Kutta condition in a careful analytic treatment of the vertex flow. Since their model does not permit reattachment, this Kutta condition becomes a dominant factor in the determination of the character of the separated flow region at all subsequent times. In contrast, the work contained herein considers the detailed flow around the vertex to be secondary to the hydrodynamics of the jets, which are assumed to be located at some finite distance from the vertex. One can speculate that for a real fluid, the initial separation predicted by Divitiis and de Socio [25] for low values of $U_{0} / W_{0}$ is followed by reattached jet flow. For those flows with larger values of $U_{0} / W_{0}$ that do not reattach, the condition of continuing flow separation is then governed by an interaction between increasing vertex velocities and diminishing jet velocities. Clearly, this is an area where more research is needed.

\section{Experimental investigation of initial water impact}

\subsection{EXPERIMENTAL SET-UP}

Drop tests, including asymmetry and horizontal impact velocity, were conducted. The objective was to investigate the fluid motion during initial water impact of a prismatic body including flow ventilation. Determination of the onset of Type B impact flow was a particular goal. The set-up consisted of a slide assembly with guide rails and linear bearings, a cart mounted to the linear-bearing slide assembly, a stiff frame connected to the cart with a symmetric prismatic wedge attached, and a free-falling weight that forced the cart along the linear-bearing slide assembly. The weight was attached to the cart by a cable through a system of pulleys that allowed the weight to fall vertically after the cart was released. The wedge ran parallel to the linear-bearing slide assembly, offset appropriately a small distance from the tank wall. The wedge could be rotated but was restrained in roll during the experiments. The slide assembly could be varied from the vertical to produce different ratios of horizontal to vertical impact velocities. The slide assembly allowed translation of the cart a maximum distance of $0.82 \mathrm{~m}$. The cart was released by an electromagnet and was forced along the slide assembly by the free-falling weight. The weight was arrested by a shock absorber shortly before the end of the slide assembly was reached. The prismatic wedge was constructed of aluminum and had 

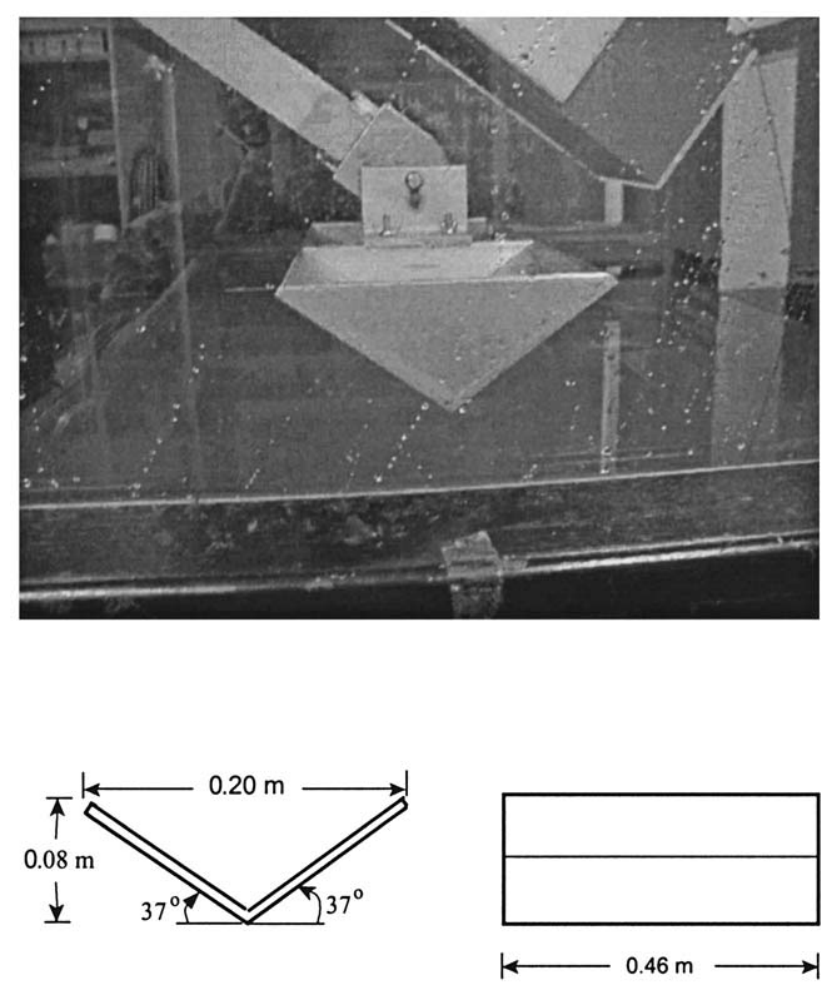

Figure 9. Photograph and schematic of drop model (wedge).

a length of $0.46 \mathrm{~m}$, a width of $0.20 \mathrm{~m}$ and elevation (i.e., deadrise) angles of $37^{\circ}$. A schematic and photograph of the model are shown in Figure 9.

Various flow visualization schemes were evaluated in order to identify the transition from Type A to Type B impact. Initially, an approach similar to the one used by Greenhow and Lin [37] was tried where a photograph of the impacting wedge was taken through a vertical glass sidewall. The clearance between the wedge end and glass should be such that the two dimensionality of the flow is preserved. However, even a small gap produces significant end flow effects. See, for example, [3, Figures 3.10-3.12] for vertical impact or Figure 10 and Figure 11 herein for oblique impact. In Figure 10, the low pressures at the vertex cause air to be drawn into a cavity on the trailing side of the wedge. This cavity was not present at the initial stages of impact, but developed from the wedge cylinder end and traveled axially along the cylinder as the wedge continued into the water. Figure 11 is a magnified view of the same conditions, again showing the air gap at the trailing upper edge with additional detail of the free surface shape unseen in Figure 10. Given the interaction of the gap hydrodynamics with the vertex hydrodynamics, it was not possible to accurately differentiate between Type A and Type B flows. For example, Figure 10 illustrates Type A flow with developing ventilation and not Type B flow with initial ventilation and subsequent re-attachment. To eliminate the uncertainties caused by the wedge edge-glass side wall gap, a vertical laser sheet located approximately one third of the wedge cylinder's length from the glass side wall was used to reduce the possibility of incorrectly identifying premature vertex ventilation during initial impact. In addition to the repeatability tests described below, the laser sheet was moved to several longitudinal locations along the wedge cylinder axis. These different longitudinal loc- 


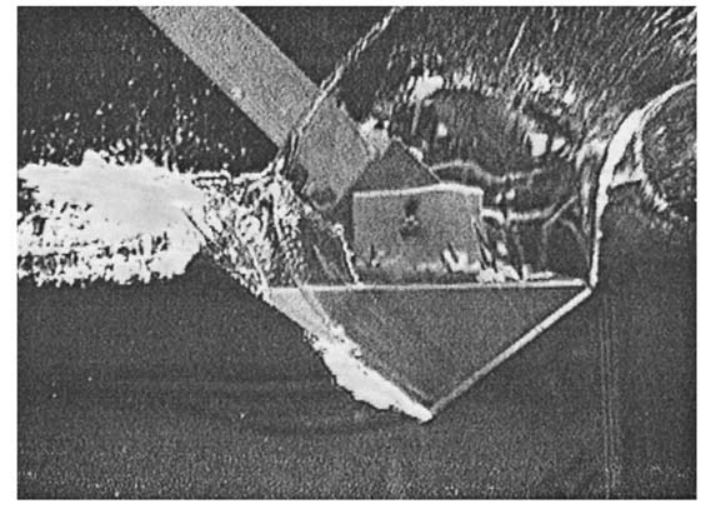

Figure 10. Type A impact and subsequent end ventilation viewed through vertical glass sidewall with oblique lighting. Note significant flow and ventilation effects between wedge end and glass. $\theta=0^{\circ}$ and $U_{0} / W_{0}=1 \cdot 0$.

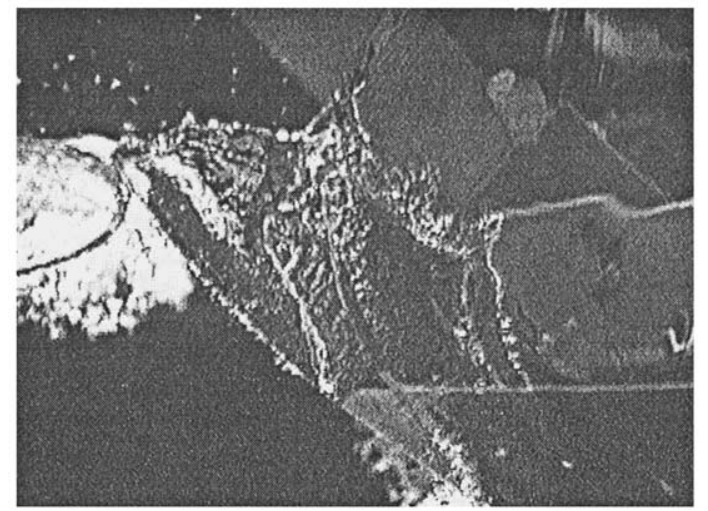

Figure 11. Magnified view of trailing wedge top during Type A impact with end ventilation. Video frame taken through vertical glass sidewall. Note flow and ventilation effects between wedge end and glass. $\theta=0^{\circ}$ and $U_{0} / W_{0}=1 \cdot 0$.

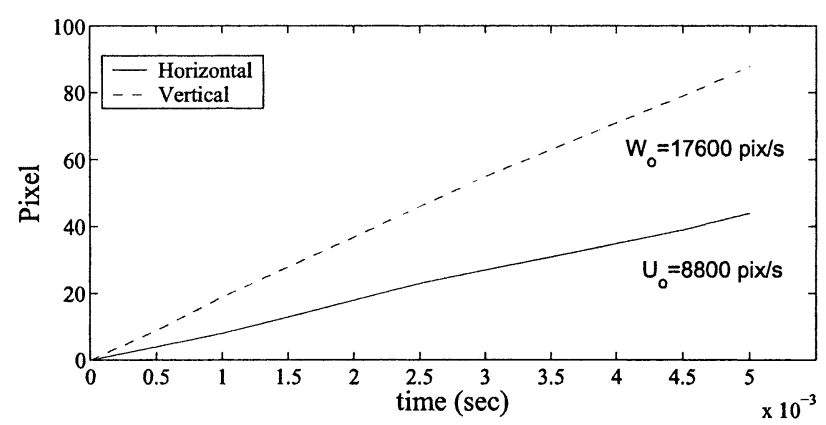

Figure 12. Distance versus time for an experiment with $U_{0} / W_{0}=0 \cdot 5$. The slopes of the lines are proportional to the impact speeds. Nominal drop speed is approximately $3 \cdot 81 \mathrm{~m} / \mathrm{sec}$.

ations produced essentially the same video images demonstrating that the flow visualizations of the early stages of impact were essentially free of end effects.

The impact was recorded using a Kodak EktaPro high speed camera at a frame rate of 2000 frames per second and affixed with a $100 \mathrm{~mm}$ lens. A Liconix argon-ion laser and attendant optics produced a light sheet perpendicular to the vertex line and approximately $0.13 \mathrm{~m}$ from the end of the body. The laser illuminated the dye-laden water and wedge. Fluorescein dye and particles, coated hollow glass spheres with mean diameter $13 \mu \mathrm{m}$, were used to visualize the impact flow and to detect the wetted surface. The velocity of the body was measured using sequential video frames. Figure 12 graphs distance versus time for a drop with a ratio of impact velocities $U_{0} / W_{0}=0.5$. The measured ratio has a standard deviation of 0.06. The wedge was dropped into quiescent water. At least three releases were conducted for every test configuration. Figures 16, 17 and 19-23 are typical examples of the video recorded experiments. 
Table 1. Experimental test configurations

\begin{tabular}{ll}
\hline$U_{0} / W_{0}$ & $\theta\left( \pm 2^{\circ}\right)$ \\
\hline $0 \cdot 0$ & $0^{\circ}, 5^{\circ}, 10^{\circ}, 15^{\circ}, 20^{\circ}, 25^{\circ}, 30^{\circ}, 34^{\circ}$ \\
0.25 & $0^{\circ}, 5^{\circ}, 10^{\circ}, 15^{\circ}, 20^{\circ}, 25^{\circ}, 30^{\circ}, 34^{\circ}$ \\
0.5 & $0^{\circ}, 5^{\circ}, 10^{\circ}, 15^{\circ}, 20^{\circ}, 25^{\circ}, 30^{\circ}, 34^{\circ}$ \\
0.75 & $0^{\circ}, 5^{\circ}, 10^{\circ}, 15^{\circ}, 20^{\circ}, 25^{\circ}, 30^{\circ}, 34^{\circ}$ \\
1.00 & $0^{\circ}, 5^{\circ}, 10^{\circ}, 15^{\circ}, 20^{\circ}, 25^{\circ}, 30^{\circ}, 34^{\circ}$ \\
1.33 & $0^{\circ}, 5^{\circ}, 10^{\circ}, 15^{\circ}, 20^{\circ}$ \\
$2 \cdot 00$ & $0^{\circ}, 5^{\circ}, 34^{\circ}$ \\
\hline
\end{tabular}

Table 2. Effective elevation (i.e., deadrise) angles determined from $\theta$ 's for drop tests

\begin{tabular}{rrl}
\hline$\theta$ & $\beta_{1}$ & $\beta_{2}$ \\
\hline $0^{\circ}$ & $37^{\circ}$ & $37^{\circ}$ \\
$5^{\circ}$ & $32^{\circ}$ & $42^{\circ}$ \\
$10^{\circ}$ & $27^{\circ}$ & $47^{\circ}$ \\
$15^{\circ}$ & $22^{\circ}$ & $52^{\circ}$ \\
$20^{\circ}$ & $17^{\circ}$ & $57^{\circ}$ \\
$25^{\circ}$ & $12^{\circ}$ & $62^{\circ}$ \\
$30^{\circ}$ & $7^{\circ}$ & $67^{\circ}$ \\
$34^{\circ}$ & $3^{\circ}$ & $71^{\circ}$ \\
\hline
\end{tabular}

Two primary observations were made of the fluid motion during impact. The first relates to the ratio of wetted points for different ratios of impact velocities and degrees of wedgeorientation asymmetry. Type A impact images show the free surface in contact with the wedge. The larger the geometric asymmetry $(\theta)$ or kinematic asymmetry $\left(U_{0} / W_{0}\right)$ the greater the difference between the intersection point and the vertex on each side of the wedge. The second primary observation relates to the parameters for onset of Type B flow and the behavior of the fluid ventilation. Type B impact images show the free surface only in contact with the wedge on one side. The free surface moves away from the wedge on the other side of the vertex. This flow detachment behaves differently depending on the degree of geometric and kinematic asymmetry.

\subsection{EXPERIMENTAL/NUMERICAL COMPARISON (TYPE A IMPACT)}

Table 1 presents the test configurations, i.e., the ratios of impact velocity and the degrees of asymmetry that were used in the experiments.

The symmetric wedge was rotated to various heel angles, $\theta$, to produce asymmetric impact and the slide assembly was set to specific angles that determined the ratio of horizontal to vertical impact velocity. The wedge was rotated at increments of $5^{\circ}$ from the symmetric position $\left(\theta=0^{\circ}\right)$ to an angle of large geometric asymmetry $\left(\theta=34^{\circ}\right)$. Table 2 gives the effective elevation (i.e., deadrise) angle on each side of the vertex for the different $\theta$ 's, where $\beta_{1}$ is the angle between the wedge body and the free surface on the right of the vertex in the video images and $\beta_{2}$ is the angle on the left.

The digital video data were recorded in memory and then transferred to computer. The image measurements were the horizontal distances between the vertex and the wetted points on each side of the body. The wetted point is defined as the location where the curved water surface intersects the body and is compared to points $B_{1}$ and $B_{2}$. For each drop, the frames following the first evidence of water contact were used to measure the wetted points and the measured $c_{2}^{*}$ 's were then averaged. Using these early frames avoided contaminating the results with end ventilation, e.g. Figure 10. Averaging over several frames reduced any significant contribution due to early-stage impact transients. The measured $c_{2}^{*}$ 's for each frame were within $15 \%$ of the final average for the smaller ratios of impact velocities and varied as much 


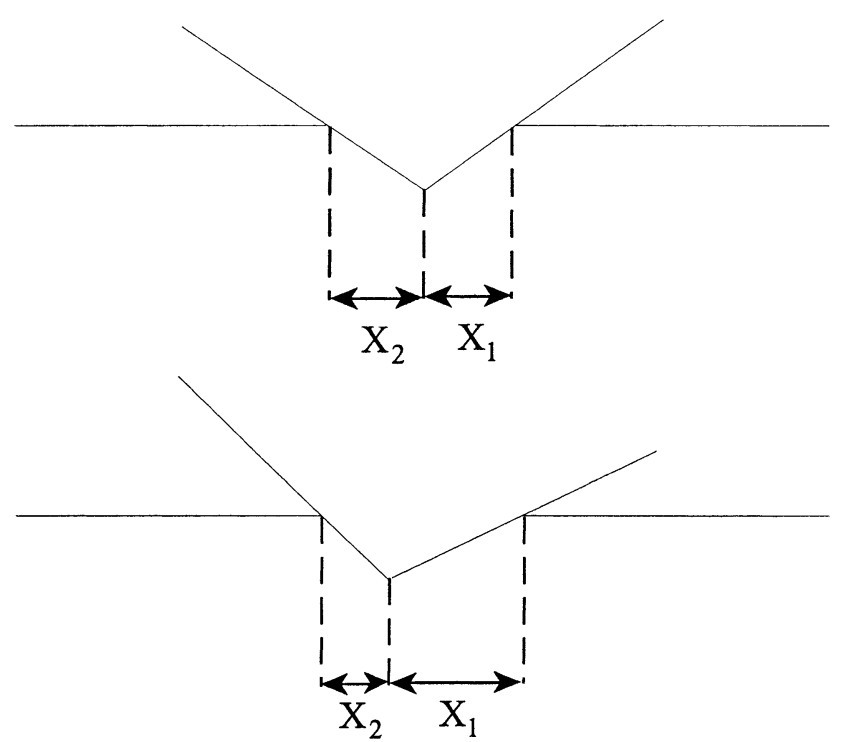

Figure 13. Locations and definitions of the intersection of the free surface for symmetric and asymmetric impact.

as $25 \%$ for the larger ratios of impact velocities. The averaged data measurements from each drop for each configuration were then averaged to obtain a representative $c_{2}^{*}$.

Figure 13 shows a symmetric and an asymmetric impact of a prismatic wedge where hydrodynamic effects are neglected. In this case, the locations of the wetted points are simply at the intersection of the undisturbed free surface and the body. This can be determined entirely by geometry and is used as a basis for comparison with the hydrodynamic effects. Let the distance from the vertex to the intersection of the undisturbed free surface on side 1 (the right side) be $X_{1}$ and the equivalent distance on side 2 (the left side) be $X_{2}$. The ratio of these two distances is defined as $d$. For a symmetric body, $d$ is equal to 1 . As the heel angle, $\theta$, increases, $d$ decreases until $\theta$ equals the elevation (i.e., deadrise) angle $\beta_{1}$ at which point, $d=0$. Figure 14 (a) shows $d$ as a function of $\theta$ for a symmetric wedge with an elevation (i.e., deadrise) angle of $37^{\circ}$. The plot of $d$ versus $\theta$ is unaffected by adding horizontal velocity since the change in $d$ is determined solely by geometry.

Hydrodynamic effects during impact create jets that move primarily along the body. Figure 14 (b) shows a graph of $c_{2}^{*}$ versus $\theta$ where the ratio of impact velocities is $U_{0} / W_{0}=0 \cdot 25$. The solid curve represents the numerical solution of the theory presented herein and the open circles the experimental data. The error bars are calculated from the standard deviation of the measured $c_{2}^{*}$ s. In this case, the addition of horizontal velocity to the impact does affect $c_{2}^{*}$ since the hydrodynamic effects are included.

The importance of the hydrodynamic effects varies depending on the type of impact. For some impacts $c_{2}^{*}$ is similar to $d$, while for other impacts, the two ratios are very different. If $B_{2}$ and $B_{1}$ are proportional to $X_{2}$ and $X_{1}$, then, despite the fact that the physical positions of the points are not the same, $d$ and $c_{2}^{*}$ would be equal. If the hydrodynamic effects are dominant, then the positions would not be proportional and $d$ and $c_{2}^{*}$ would be very different.

Graphs of $d-c_{2}^{*}$ as a function of asymmetry and horizontal impact velocity are shown in Figure 15. The dashed curve represents the geometric prediction, the solid curve the numerical, and the open circles the experimental data. When there is no horizontal velocity, simply vertical impact with asymmetry (Figure 15 a), there are only small differences between the 

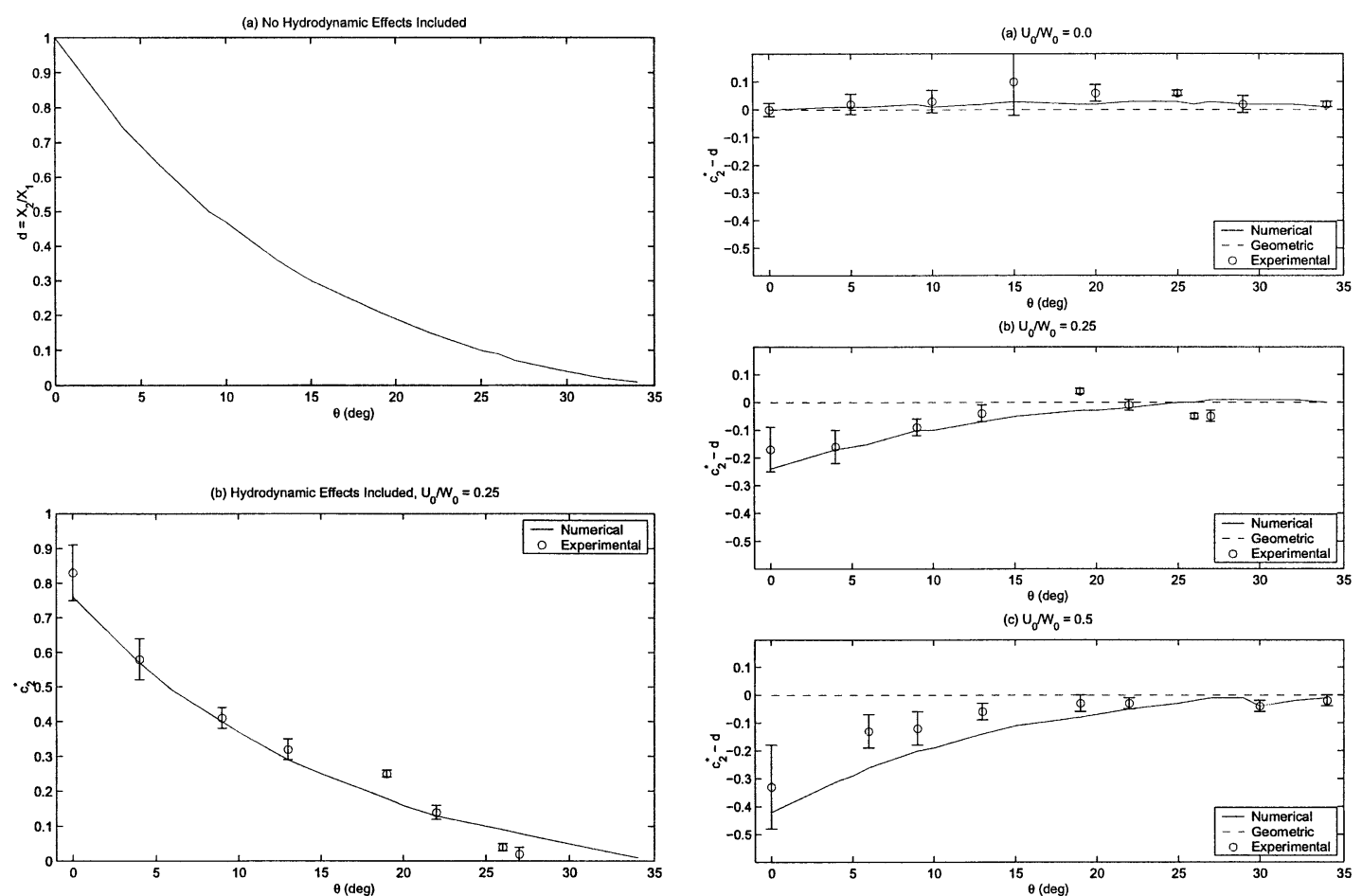

Figure 14. (a) Graph of the ratio, $X_{2}$ to $X_{1}$, for asymmetric impact (effect of geometry, no hydrodynamic effects). (b) Graph of the ratio, $B_{2}$ to $B_{1}$, for a ratio

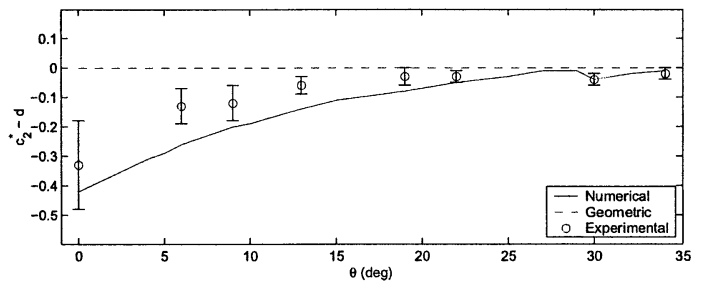

Figure 15. The difference between $c_{2}^{*}$ and $d$ predicted and the experimental data. of impact velocities $U_{0} / W_{0}=0 \cdot 25$.

predictions for $c_{2}^{*}$, the experimental results for $c_{2}^{*}$, and the geometric ratio $d$. The numerical solution captures the slight curve in the experimental data; however, in this type of impact the asymmetry dominates the flow and the geometric prediction gives reasonable results.

Generally, when $\theta$ is large, the geometric asymmetry dominates the flow and $c_{2}^{*}$ is very close to $d$. When $\theta$ is small and there is horizontal impact velocity, the hydrodynamics become very important and $c_{2}^{*}$ diverges from $d$.

Typical video frames of Type A impact are presented in Figures 16 and 17. The physical dimensions represented by the images are $0.05 \mathrm{~m}$ vertical by $0.15 \mathrm{~m}$ horizontal. The body contour lines have been superposed, while the curved free surface visualizations are due to the reflection from the seed particles in the water. The frames are sequential $(\Delta t=0.5 \mathrm{msec})$ and illustrate how the jets move along the body as the impact progresses.

\subsection{InVESTIGATION OF INITIAL FLOW VENTILATION (TYPE B IMPACT)}

Flow ventilation occurs when the liquid surface detaches from the body on one side. When this occurs the difference in forces from pressure on each side of the body becomes extreme [1]. Since this phenomenon can occur suddenly, it is important to predict the parameter ranges 

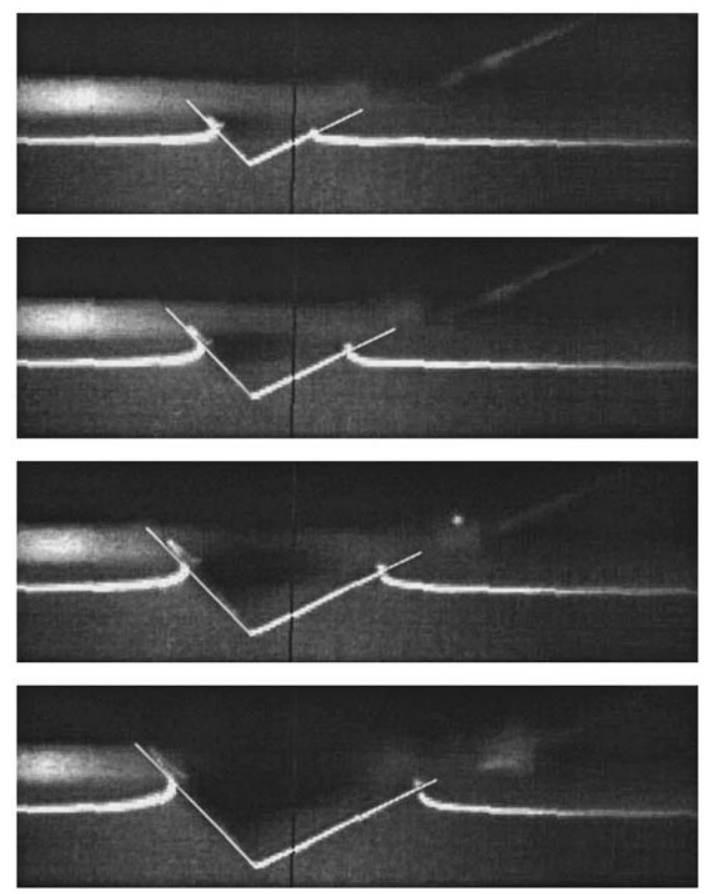

Figure 16. Type A impact with $\theta=10^{\circ}, U_{0} / W_{0}=$ 0.25 , and $\Delta t=0.5 \mathrm{msec}$.
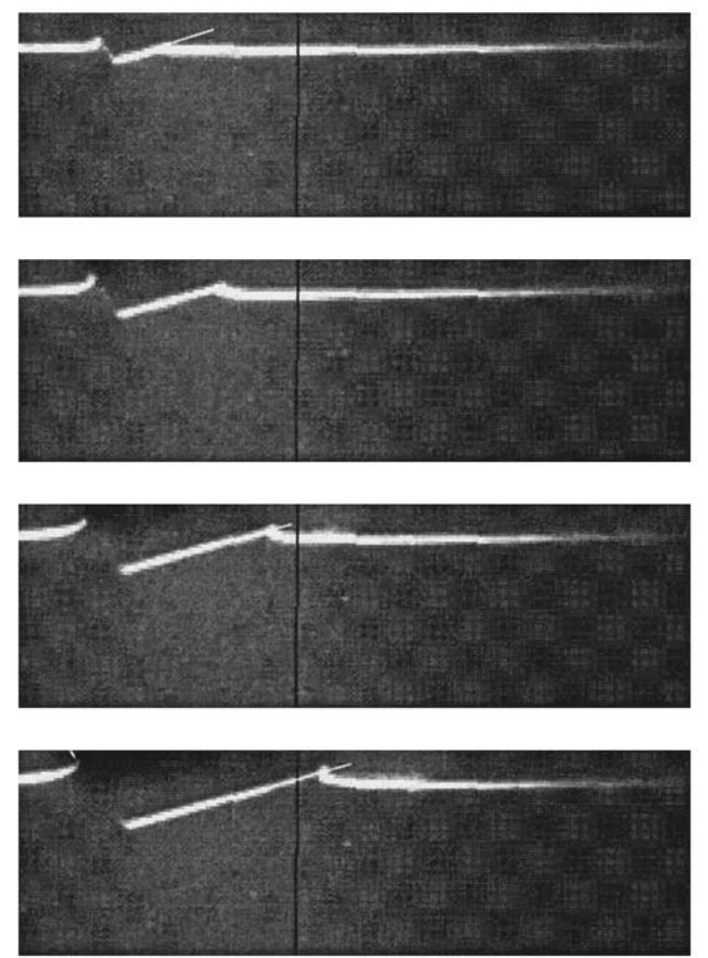

Figure 17. Type A impact with $\theta=20^{\circ}$ and $U_{0} / W_{0}=$ 0.5 .

where this would occur. Figure 18 shows $c_{2}^{*}$ versus $\theta$ for an impact with ratios of 0.5 and 0.75 for horizontal to vertical impact velocity.

This configuration is of interest because ventilation did not occur for the symmetric case, but did occur before the maximum $\theta$ was reached. In Figure 18 ventilation is indicated by $c_{2}^{*}$ becoming zero. For $U_{0} / W_{0}=0.75$, the theory predicts ventilation at a lower angle of asymmetry than was determined from the experiment, as is true generally. The theory closely matches the initial $\theta$ value for $U_{0} / W_{0}=0.50$ and most other values as well. Table 3 compares the theoretical predictions for ventilation with the ventilation limits measured experimentally. The incremental change in experimental $\theta$ is $5^{\circ}$. Table 3 is to the nearest $5^{\circ}$. Overall, the theory produces reasonable values when compared to experiments.

To study the transition from Type A to Type B flow, video was recorded of impacts for increasing $\theta$ at a fixed velocity ratio. Figure 19 shows images taken at approximately the same impact time for different $\theta$ 's at a velocity ratio of $U_{0} / W_{0}=0.75$. For $\theta=15^{\circ}$ the flow is smooth along the body exhibiting similarity flow type behavior, and there is no ventilation. For $\theta$ of $20^{\circ}$ the flow is still attached, but a separation cavity is beginning to form at the vertex. The jet remains attached to the body, however, so this is still a Type A impact. For $\theta$ greater than $20^{\circ}$ the flow separates from the body at the vertex and the jet does not re-attach. Increasing the asymmetry makes these effects more obvious. Since similarity type flow requires that the flow looks the same over time, the transition region at initial impact but prior to ventilation is no longer similar. Some video records show that for Type B flow, the shape of the free surface 

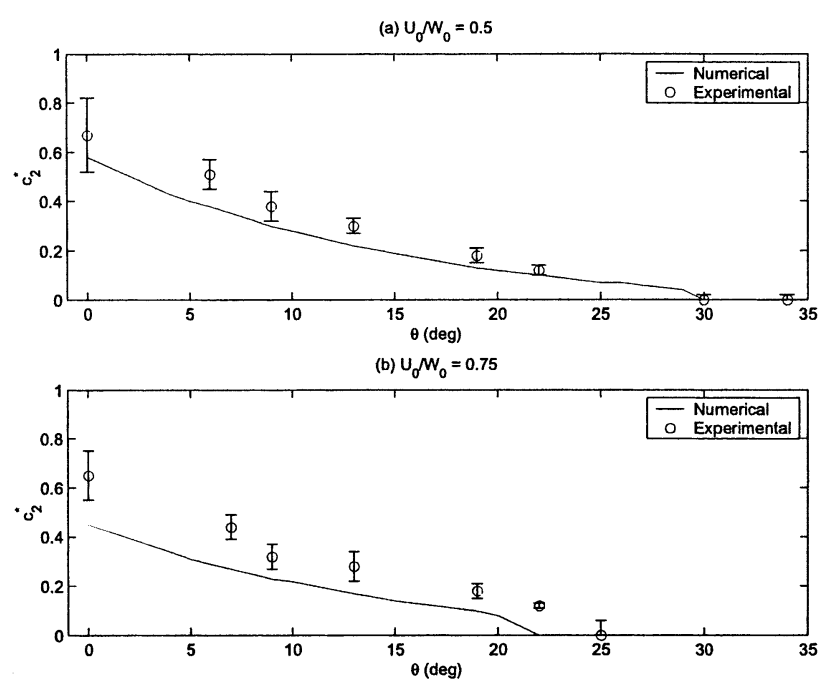

Figure 18. $c_{2}^{*}$ versus $\theta$ for (a) $U_{0} / W_{0}=0.5$ and (b) $U_{0} / W_{0}=0.75$.

Table 2. Experimental and theoretical predictions for initiation of ventilation

\begin{tabular}{lcc}
\hline \multicolumn{2}{l}{$U_{0} / W_{0} \theta_{\text {VENT }}($ Experimental, $\Delta \theta$ VENT } & $\left.=5^{\circ}\right) \theta_{\text {VENT }}$ (Theoretical) \\
\hline 0.5 & $30^{\circ}$ & $30^{\circ}$ \\
0.75 & $25^{\circ}$ & $20^{\circ}$ \\
1.0 & $15^{\circ}$ & $10^{\circ}$ \\
1.33 & $5^{\circ}$ & $5^{\circ}$ \\
2.0 & $0^{\circ}$ & $0^{\circ}$ \\
\hline
\end{tabular}

evolves with time, e.g. Figure 20. These Figures are in temporal order, but are not sequential video frames. Therefore, Type B flow cannot be assumed to be similarity type flow even after the flow has detached from the body.

The behavior of the flow as the wedge impacts the water depends on the degree of asymmetry and the amount of horizontal impact velocity. When ventilation is initiated, the flow, initially attached to the body, separates. As the jet on the left side separates from the body, the flow around the vertex also moves off the body (see Figure 21). This time delay prior to ventilation is not predicted by the present theory. The theory assumes similarity flow when determining the initial conditions. In particular, the determination of the wetted points is based on the liquid already being in motion. In reality the water accelerates from rest. The importance of fluid compressibility for accelerating fluid has been discussed by others, e.g. [16], [17], or [18]. The model presented here does not take compressibility into account and thus relies upon the assumptions of similarity during the first instants of impact. The video images for many Type B impacts show attached flow for only the first one or two frames. The vertical distance traversed by the body is on the order of $2.0 \mathrm{~mm}$ and the time is less than one msec. As the Type B impact continues the flow exhibits highly non-similar behavior near the vertex. In some cases the free surface forms a jet that initially moves along the body and then detaches, but remains jet-like (see Figure 21). In other cases the free surface does not form a 

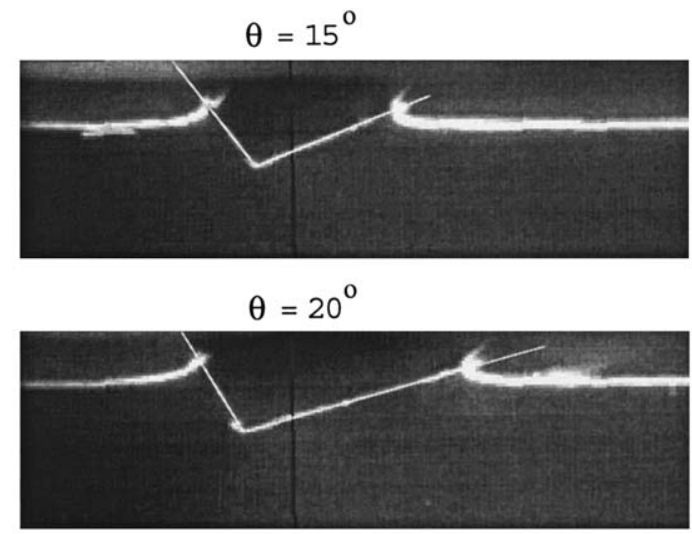

$\theta=25^{\circ}$

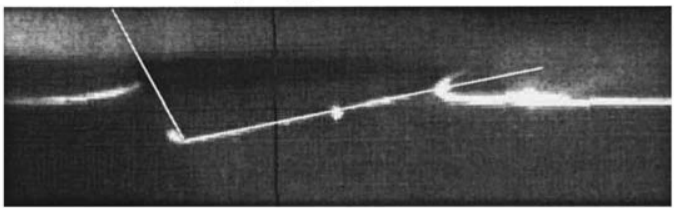

$\theta=30^{\circ}$

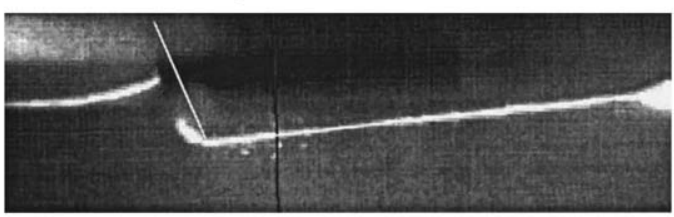

$\theta=34^{\circ}$

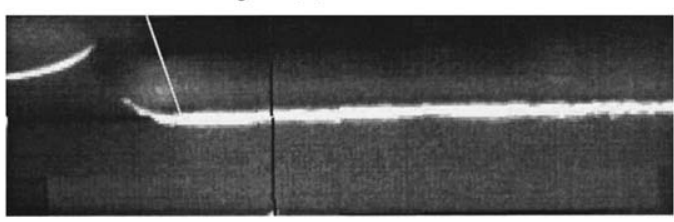

Figure 19. Transition from Type A to Type B impact for several $\theta$ 's and $U_{0} / W_{0}=0.75$.
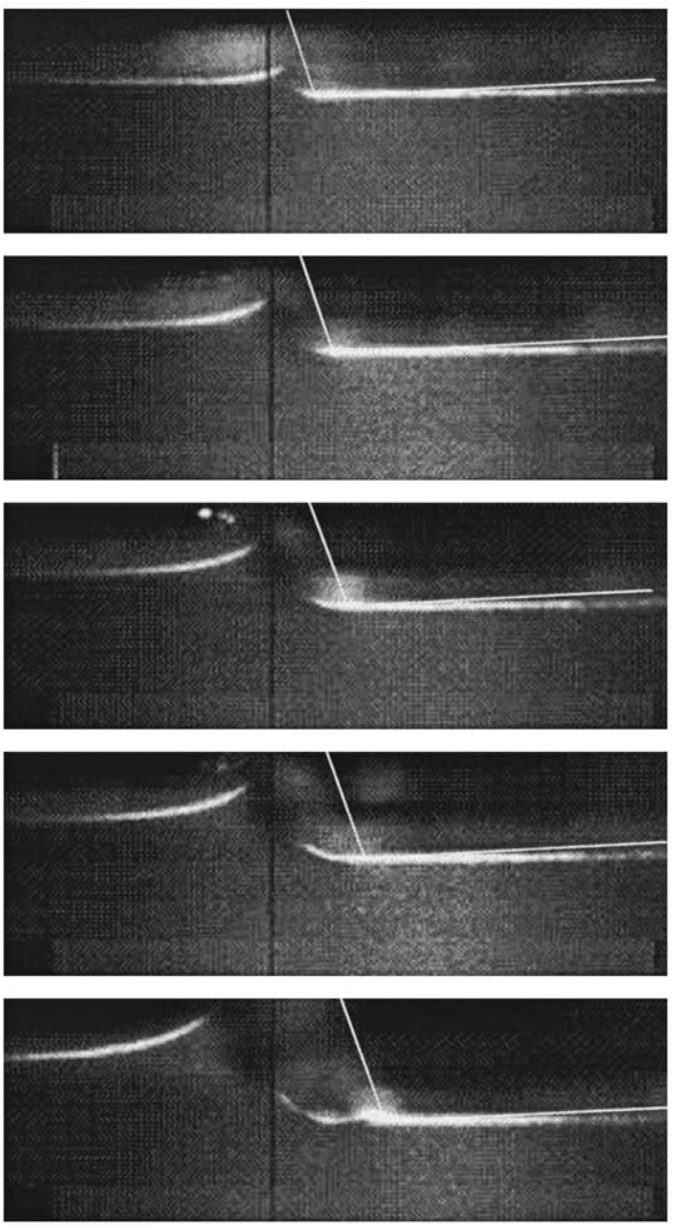

Figure 20. Type B flow showing evolution of free surface for impact with $\theta=34^{\circ}$ and $U_{0} / W_{0}=1 \cdot 0$.

jet and remains horizontal as the body moves away (see Figure 22). The left side flow near the vertex curves from the body. Near the jet the connection between the flow curving from the vertex and the free surface often becomes indistinct. The reason is not clear since the air-water mixing and local spray prevent a clear image using the laser sheet.

Of special interest is small asymmetry and large horizontal impact velocity. In this situation ventilation is due mainly to the horizontal velocity component. Figures 22 and 23 show such an example. Figure 23, an extension in time of Figure 22, shows a fully developed Type B impact. The images in Figure 23 are in temporal order, but are not sequential frames. Initially the flow appears to be attached, but quickly separates near the vertex. The free surface on the left side never forms a jet since the ventilation is caused by the body moving quickly to the right. 

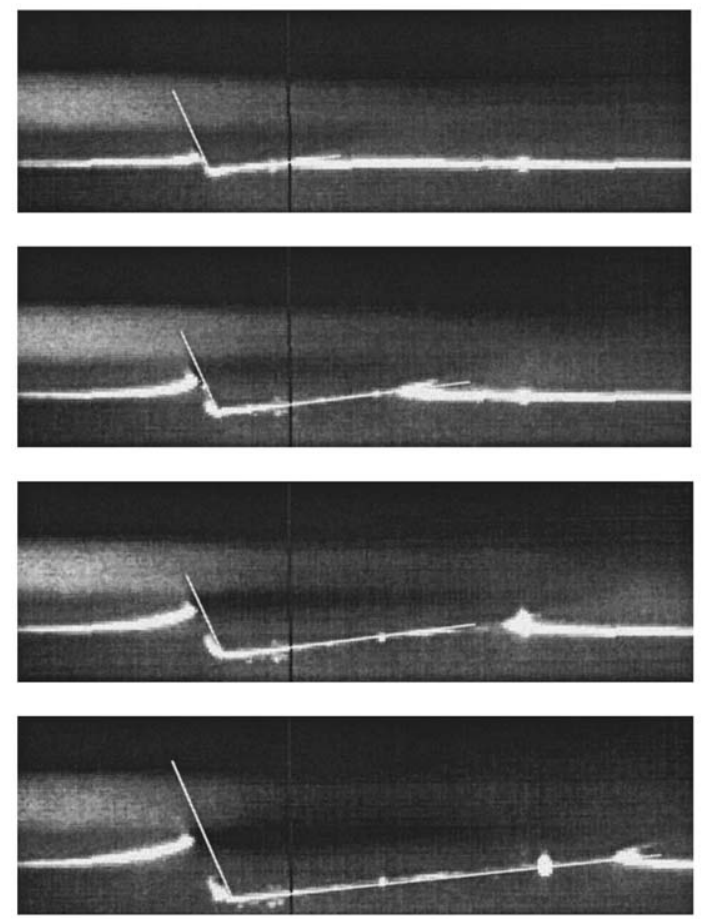

Figure 21. Type B impact with $\theta=30^{\circ}$ and $U_{0} / W_{0}=$ 0.75 .
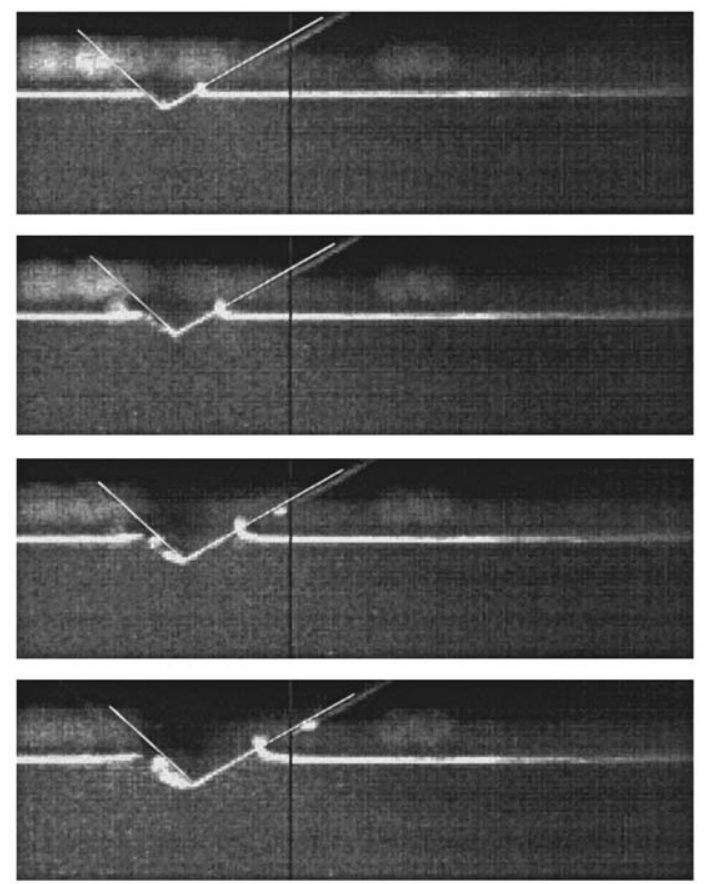

Figure 22. Type B impact with $\theta=5^{\circ}$ and $U_{0} / W_{0}=$ $2 \cdot 0$

\section{Concluding remarks}

The relevant details of the impact flow as well as jet-formation are captured by the model presented here. Initial separation of the flow from the vertex due to asymmetric-impact or horizontal-impact velocity is examined in relation to the present theory. Two-dimensional vortex distributions with time-dependent free vortex shedding are used to model the boundary value problem. The impact model described herein is capable of determining hydrodynamic characteristics, including the transient impact loads, for a wedge impacting with asymmetry and horizontal-impact velocity. See [1] for the pressures, forces, and moments associated with various impact configurations.

Experimental drop tests of a prismatic wedge were performed to increase understanding of initial water-impact physics when asymmetry and horizontal-impact velocity are present. In particular, the initiation of Type B flow was investigated. The theoretical predictions of $c_{2}^{*}$ were compared to experimental results. The ratios of the jet spray-root location, $c_{2}^{*}$, predicted were compared with the experimental data. Good agreement between the data and the numerical predictions was shown for small degrees of asymmetry and small ratios of horizontal to vertical impact velocity. The numerical prediction of the transition point from Type A to Type B flow compared very well with the experimental observations. The flow behavior just before and at ventilation is very complex and is not incorporated in this impact model. This is clearly an area requiring additional research. This experimental investigation of initial flow detachment from the vertex revealed many interesting flow characteristics. Several of these 

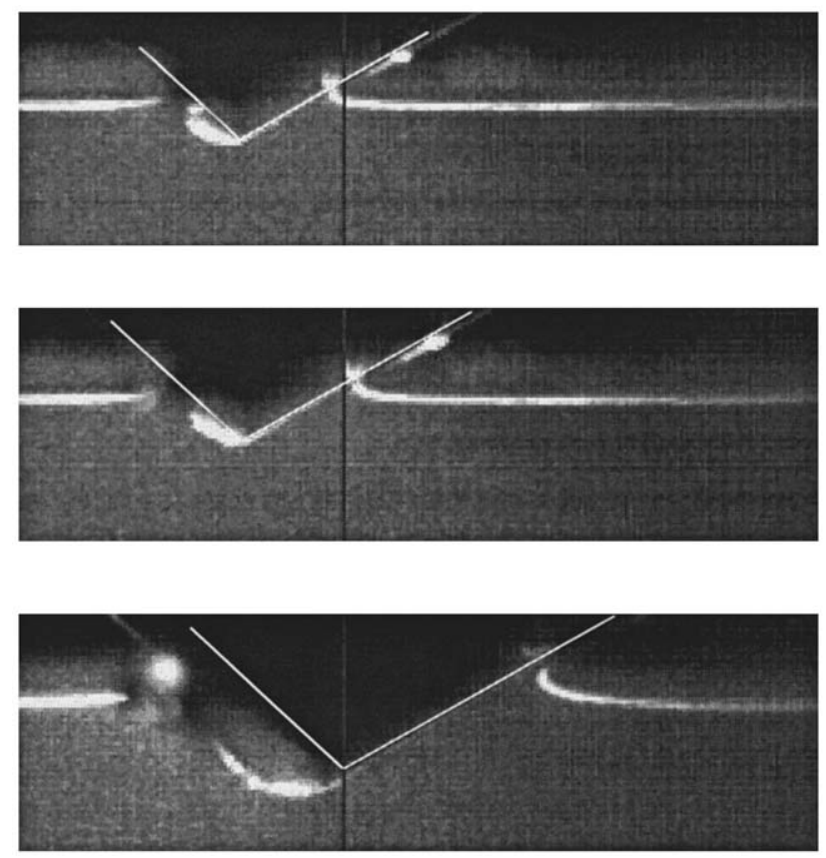

Figure 23. Fully developed separated flow during impact for $\theta=5^{\circ}$ and $U_{0} / W_{0}=2 \cdot 0$.

characteristics are not entirely understood or explained by the existing theory. Further study of this flow and the development of a flow model would be an important step in understanding impact with asymmetry and horizontal impact velocity.

\section{Acknowledgements}

This research has been supported by the Department of Defense, Office of Naval Research and the Link Foundation, and is part of a fundamental study conducted at the University of Michigan, Ann Arbor, on nonlinear impact loads, extreme vessel motion and high-speed planing hull technology. The drop tests described here were conducted in the Marine Hydrodynamics Laboratory at the University of Michigan, Ann Arbor with the help of Luke DeVito, Kevin Maki and Joe Krasny.

\section{References}

1. C.Q. Judge, A Theory for Asymmetric Vessel Impact with Horizontal Impact Velocity. PhD thesis, University of Michigan, Ann Arbor, Michigan (2000) 218 pp.

2. T. von Kármán, The Impact of Seaplane Floats During Landing. Technical Note 321 NACA, Washington, D.C. (1929).

3. H. Wagner, Über Stoss und Gleitvorgänge an der Oberfläche von Flüssigkeiten. ZAMM 12 (1932) 193-215.

4. J. Sydow, Über den Einfluss von Federung und Kielung auf den Landestoss. Jarhbuch der deutschen Luftfarhtforschung (1938) 329-338.

5. W.L. Mayo, Analysis and Modification of Theory for Impact of Seaplanes on Water. Technical Note 1008 NACA, Washington, D.C. (1945).

6. R.L. Blisplinghoff and C.S. Dohery, Some studies of the impact of vee wedges on a water surface. J. Franklin Inst. 253 (1952) 547-562. 
7. Z.N. Dobrovol'skaya, On some problems of similarity flow of fluid with a free surface. J. Fluid Mech. 36 (1969) 805-829.

8. R. Cointe and J.-L. Armand, Hydrodynamic impact analysis of a cylinder. J. Offshore Mech. Artic Eng. 109 (1987) 237-243.

9. R. Cointe, Two-dimensional water-solid impact. J. Offshore Mech. Arctic Eng. 111 (1989) 109-114.

10. R. Cointe, Free-surface flows close to a surface-piercing body. In: T. Miloh (ed.), Mathematical Approaches in Hydrodynamics. Philadelphia: Soc. Industr. Appl. Math. (1991) pp. 319-334.

11. S.D. Howison, J.R. Ockendon and S.K. Wilson, Incompressible water-entry problems at small deadrise angles. J. Fluid Mech. 222 (1991) 215-230.

12. M. Greenhow, Wedge entry into initially calm water. Appl. Ocean Res. 9 (1987) 214-223.

13. R. Zhao and O. Faltinsen, Water entry of two-dimensional bodies. J. Fluid Mech. 246 (1993) 593-612.

14. R. Zhao, O. Faltinsen and J. Aarsnes, Water entry of arbitrary two-dimensional sections with and without flow separation. In: Proc. $21^{\text {st }}$ Symp. Naval Hydrodyn. Trondheim, Norway: National Academy Press (1997) pp. $408-423$.

15. S. Muzaferija, M. Peric, P. Sames, and T. Schellin, A two-fluid Navier-Stokes solver to simulate water entry. In: Proc. $22^{\text {nd }}$ Symp. Naval Hydrodyn. Washington D.C.: National Academy Press (1998) pp. 638-651.

16. A.A. Korobkin, Water impact problems in ship hydrodynamics. In: M. Ohkusu (ed.), Water Impact Problems in Ship Hydrodynamics. Southampton: Computational Mechanics Publications (1996) pp. 323-371.

17. A.A. Korobkin, Asymptotic theory of liquid-solid impact. Phil. Trans.: Math. Phys. Eng. Sci. 35 (1997) 507-522.

18. E.F. Campana, A. Carcaterra, E. Ciappi and A. Iafrati, Parametric analysis of slamming forces: compressible and incompressible phases. In: H. Kim, S.H. Lee and S.J. Lee (eds.), Proc. $3^{\text {rd }}$ Int. Conf. Hydrodyn. ICHD98. Seoul, Korea (1998) pp. 167-172

19. D. Savitsky, R.E. Prowse and D.H. Lueders, High-Speed Hydrodynamic Characteristics of a Flat Plate and 20 Degree Dead-Rise Surface in Unsymmetrical Planing Conditions. Technical Note 4187, NACA, Washington, D.C. (1953).

20. A.A. Korobkin, Inclined entry of a blunt profile into an ideal fluid. Fluid Dynamics 23 (1988) 443-447.

21. T. Miloh, On the oblique water-entry problem of a rigid sphere. J. Eng. Math. 25 (1991) 77-92.

22. P.R. Garabedian, Oblique water entry of a wedge. Comm. Pure Appl. Math. VI (1953) 157-165.

23. B.S. Chekin, The entry of a wedge into an incompressible fluid. J. Appl. Math. Mech. 53 (1989) 300-307.

24. A. Iafrati, Hydrodynamics of asymmetric wedges impacting the free surface. In: E. Oñate (ed.), Eur. Congr. Comp. Methods Appl. Sci. Eng. ECCOMAS Barcelona, Spain (2000) (on CDrom) pp. 1-13.

25. N. de Divitiis and M.L. de Socio, Impact of floats on water. J. Fluid Mech. 471 (2002) 365-379.

26. M.P. Tulin, The theory of slender surfaces planing at high speeds. Schiffstechnik 4 (1957) 125-133.

27. W.S. Vorus, An extended slender body model for planing hull hydrodynamics. In: SNAME Great Lakes and Great Rivers Section Meeting. Cleveland, Ohio. Jersey City (NJ): SNAME (1992).

28. W.S. Vorus, A flat cylinder theory for vessel impact and steady planing resistance. J. Ship Res. 40 (1996) 89-106.

29. L. Xu, A.W. Troesch and W.S. Vorus, Asymmetric vessel impact and planing hydrodynamics. J. Ship Res. 42 (1998) 187-198.

30. B.R. Savander, S.M. Scorpio and R.K. Taylor, Steady hydrodynamic analysis of planing surfaces. J. Ship Res. 46 (2002) 248-279.

31. L. Xu, A Theory for Asymmetrical Vessel Impact and Steady Planing. PhD thesis, University of Michigan, Ann Arbor, Michigan (1998) 128 pp.

32. E. Fontaine and R. Cointe, Asymptotic theories of incompressible water entry. In: Proceedings of the NATO/AGARD Symposium on High Speed body motion in water. Kiev. NATO/AGARD (1997) 25-1-25-8.

33. R. Peterson, D. Wyman and C. Frank, Drop Tests to Support Water-Impact and Planing Boat Dynamics Theory. CSS Technical Note TR-97, Coastal Systems Station, Panama City, FL. (1997).

34. N.I. Muskhelishvili, Singular Integral Equations. Leyden: Noordhoff International Publishing (1953) 447 pp.

35. F.G. Tricomi, Integral Equations. New York: Interscience (1957) 238 pp.

36. E. Fontaine and R. Cointe, A second-order solution for the wedge entry problem with small deadrise angle. In: Proc. 7th Int. Workshop Water Waves Floating Bodies. Val de Reuil 111 (1992) pp. 105-109.

37. M. Greenhow and W.-M. Lin, Nonlinear Free Surface Effects: Experiments and Theory. Report No. 83-19 Cambridge, Massachusetts: Dept. Ocean Eng., Mass. Inst. Technol. (1983). 